\title{
1 Genome of the estuarine oyster provides insights into climate impact
}

3

\section{and adaptive plasticity}

\author{
Subtitle: Insights into climate impact and adaptive plasticity
}

Ao $\mathrm{Li}^{1,2,3 \dagger}$, He $\mathrm{Dai}^{4 \dagger}$, Ximing Guo ${ }^{5 \dagger}$, Ziyan Zhang, ${ }^{1,6}$, Kexin Zhang ${ }^{1,6}$, Chaogang Wang ${ }^{1,6}$, Wei Wang ${ }^{1,3,7}$, Hongju Chen ${ }^{4}$, Xumin $\mathrm{Li}^{4}$, Hongkun Zheng ${ }^{4}$, Guofan Zhang $^{1,3,7^{*}}$ and $\mathrm{Li} \mathrm{Li}^{1,2,3^{*}}$

${ }^{1}$ CAS and Shandong Province Key Laboratory of Experimental Marine Biology, Center for Ocean Mega-Science, Institute of Oceanology, Chinese Academy of Sciences, Qingdao, China. ${ }^{2}$ Laboratory for Marine Fisheries Science and Food Production Processes, Pilot National Laboratory for Marine Science and Technology, Qingdao, China. ${ }^{3}$ National and Local Joint Engineering Key Laboratory of Ecological Mariculture, Institute of Oceanology, Chinese Academy of Sciences, Qingdao, China. ${ }^{4}$ Biomarker Technologies Corporation, Beijing, China. ${ }^{5}$ Haskin Shellfish Research Laboratory, Department of Marine and Coastal Sciences, Rutgers University, Port Norris, NJ, USA. ${ }^{6}$ University of Chinese Academy of Sciences, Beijing, China. ${ }^{7}$ Laboratory for Marine Biology and Biotechnology, Pilot National Laboratory for Marine Science and Technology, Qingdao, China. 'These authors contributed equally: A. Li, H. Dai and X. Guo. *e-mail: $\underline{\text { lili@qdio.ac.cn and gzhang@qdio.ac.cn }}$ 


\section{Abstract}

23 Understanding the roles of genetic divergence and phenotypic plasticity in adaptation

24 is central to evolutionary biology and important for assessing adaptive potential of 25 species under climate change. Analysis of a chromosome-level assembly and 26 resequencing of individuals across wide latitude distribution in the estuarine oyster 27 (Crassostrea ariakensis) revealed unexpectedly low genomic diversity and population 28 structures shaped by historical glaciation, geological events and oceanographic forces. 29 Strong selection signals were detected in genes responding to temperature and salinity 30 stress, especially of the expanded solute carrier families, highlighting the significance 31 of gene expansion in environmental adaptation. Genes exhibiting high plasticity 32 showed strong selection in upstream regulatory regions that modulate transcription, 33 indicating selection favoring plasticity. Our findings suggest that genomic variation 34 and population structure in marine bivalves are heavily influenced by climate history 35 and physical forces, and gene expansion and selection may enhance phenotypic 36 plasticity that is critical for the adaptation to rapidly changing environments. 
38

Climate change poses a serious threat to global biodiversity and the stability of ecosystems. Oceans bear the blunt of climate change as they absorb most of the energy and about one-third of the carbon dioxide created by human activities (1). World oceans become warmer and more acidic. Sea levels are rising, and ocean circulation patterns are changing. With the rising of global temperature, dry regions are getting drier, while wet regions become wetter (2), increasing salinity difference among estuaries (3). These rapid changes in world's oceans are having a significant impact on marine ecosystems, much of which remains poorly understood. The impact may vary among different organisms, and changes in keystone species may have a large effect on stability of an ecosystem. Understanding how organisms adapt to environmental shifts is fundamental to evolutionary biology and critical in assessing their adaptive potential under climate change.

The ability of an organism to survive and adapt to sudden environmental changes depends on available genetic variation and phenotypic plasticity. The standing genetic variation of a species is the outcome of its evolutionary history shaped by mutation, selection and genetic drifts (4-6). Past and on-going climate and geological events may leave signatures of selection or bottleneck in the genome and influence the geographic distribution of genetic variation (7-9). Many recent studies have revealed the significance of phenotypic plasticity in environmental adaptation (10-15). Phenotypic plasticity may buffer against sudden environmental changes and provides time for adaptation to occur $(10,13,14,16)$. Phenotypic plasticity is particularly pronounced and important for sessile organisms inhabiting estuarine and intertidal zones that cannot use avoidance to cope with wide fluctuations in environmental conditions $(10,11,17)$, although the genetic basis of the enhanced plasticity is not clear $(18,19)$.

Oysters are keystone species in coastal and estuarine ecology providing critical ecological services as filter-feeders and habitat engineers. As sessile bivalves thriving in the coastal zone, oysters are well adapted to highly dynamic environmental conditions, as reflected in their high genetic diversity and plasticity (10, 20-23), 
67 making them interesting models for studying responses to climate change. In addition

68 to threats from climate change, oyster populations worldwide have been decimated by 69 over-fishing, habitat destruction and infectious diseases $(24,25)$. The estuarine 70 (Jinjiang or Suminoe) oyster (Crassostrea ariakensis, Fig. 1a) is broadly distributed in 71 estuaries of East Asia over large latitude and experiences wide ranges of temperature 72 and salinity (26-28). Unlike the Pacific oyster (Crassostrea gigas), a sympatric 73 sister-species that is highly abundant in high-salinity waters of higher latitude, the 74 estuarine oyster is only found in estuaries at low abundance within its wide 75 distribution range. The evolutionary pathways leading to the stark differences in 76 abundance, distribution and salinity preference are not clear but important to our 77 understanding of adaptive evolution.

78 While genetic changes can be inferred with genetic markers, whole-genome analysis 79 is essential for exploring all genetic variation and identifying genes and selection 80 events that are critical for adaption. Using multi-omic analyses, we previously showed 81 that the Pacific oyster has a highly polymorphic genome with extensive expansion of 82 environment-responsive genes, and plasticity is positively correlated with local 83 adaptation $(10,22)$. In this study, we produced a chromosome-level assembly of the 84 estuarine oyster genome, re-sequenced 264 wild oysters collected from 11 estuaries, 85 and conducted transcriptomic studies of environmental response to understand its 86 genetic variation, population structure, phenotypic plasticity and genomic signatures 87 of selection or bottleneck that may be linked to environmental changes. Our results 88 show that the estuarine oyster has significantly lower genetic diversity than its 89 sister-species probably due to the impact of past glaciation on its unique estuarine 90 lifestyle. Its population structure is heavily influenced by geological events and ocean 91 currents. Expansion and selection in regulatory regions of environment-responsive 92 genes may enhance phenotypic plasticity that is critical for the adaptation to rapidly 93 changing environments. 
94

95

96

\section{Results and Discussion}

Genome assembly and annotation. We produced a chromosome-level assembly of the estuarine oyster genome of an estimated size of $583.42 \mathrm{Mb}$ (fig. S1, table S1), using a combination of nanopore long-reads (184 Gb), Illumina paired-end short-reads (64 Gb) and $\mathrm{Hi}-\mathrm{C}(106 \mathrm{~Gb})$ sequences and a hierarchical assembly approach. The final assembly consisted of 630 contigs with a N50 of $6.97 \mathrm{Mb}$, spanning $613.89 \mathrm{Mb}, 99.6 \%$ of which or 416 scaffolds with a scaffold N50 of 62.26 $\mathrm{Mb}$ were assembled into 10 linkage groups corresponding to the haploid chromosome number (Fig. 1b and c, table S2). To our knowledge, this is the most contiguous assembly produced with nanopore long-read sequencing for a bivalve mollusc (29-31). Over $97.9 \%$ of genomic short-reads and $97.2 \%$ RNA-seq reads were mapped to the assembly that also captured $92.54 \%$ of the Benchmarking Universal Single Copy Orthologs (BUSCO) datasets (Fig. 1d, table S3), indicating the assembly has a high level of completeness. The accuracy of genome sequence was $98.32 \%$ as compared with Sanger sequencing (table S4). These results indicate that the estuarine oyster genome assembly is of high quality and can be used for down-stream analyses.

The estuarine oyster genome encoded 29,631 protein-coding genes as indicated by homology, de novo prediction and mRNA transcripts, and $96.13 \%$ of which were functionally annotated (table S5). Various non-coding RNA sequences were also identified and annotated in the genome, including 1,077 transfer RNAs, 20 microRNAs and 131 ribosomal RNAs. A total of $332.40 \mathrm{Mb}(54.14 \%)$ of repetitive elements were identified (table S6), which is higher than the $43 \%$ observed in the Pacific oyster (32). Gene density was inversely correlated to the repetitive element content across all chromosomes (fig. S2, $\rho=-0.308, p<0.001$ ). Genome-wide polymorphism was $0.58 \%$, which is less than half as that of the Pacific oyster (22), probably due to population bottleneck caused by past glaciations more severely impacting its estuarine lifestyle (see below).

Genome resequencing, variation calling and population structure. We generated 
3.81 Tb clean whole-genome resequencing data from 264 wild oysters collected from 11 estuaries covering most of the distribution range $(26,27)$ (Fig. 2a). The genome mapping rate averaged $95.3 \%$ varied from $86.5 \%$ to $96.7 \%$, and the mapped read depth averaged 19.89x. The data generated 145,271,754 SNPs (ranging from 487,881 to 640,962 per individual) and $103,080,822$ indels (ranging from 342,486 to 443,381 per individual). Overall, there were 0.47 heterozygous SNPs per Kb per individual (table S7), which was $~ 35$-fold lower than that in Pacific oyster populations (10).

Structure analysis of genome-wide SNPs revealed significant differentiation among different geographic populations, consistent with previously analyses of fitness-related traits, neutral markers and transcriptomic data (26, 33-35), but with higher resolution. The optimal number of population clusters was identified as $k=3$ (fig. S3), representing three main regions along the coast of northern China (NC, 5 sites), middle China (MC, 2 sites) and southern China (SC, 4 sites) (fig. S4). Principle component analysis (PCA), explaining $16 \%$ of genetic variance by two PCs, consistently revealed three distinct populations corresponding to $\mathrm{NC}, \mathrm{MC}$ and SC. Further, fine-scale subpopulations were detected where oysters from Qingdao (QD) and southern subpopulation (SC-b) including Taishan (TS) and Qinzhou (QZh) were separated from NC and SC, respectively (Fig. 2b). Moreover, phylogenetic analysis using neighbor-joining (NJ) method supported PCA clustering, by first distinguishing southern populations from others and then identified another subpopulation (NC-a) within NC located in estuaries of southern Bohai Sea including Binzhou (BZ) and Dongying (DY) (Fig. 2c). A total of six subpopulations were identified for 11 estuaries, greatly improving the resolution of the fine-population structure. Pairwise $F_{\mathrm{ST}}$ using all polymorphic positions revealed exceptionally high divergence between SC and other populations, ranged from 0.143 to 0.225 (Fig. 2d), while divergence within SC, MC and NC populations was much lower (table S8). Oysters from MC and $\mathrm{NC}$ were clustered together in phylogenetic tree with a lower genetic divergence $\left(F_{\mathrm{ST}}\right.$ $<0.05)$, which is comparable with the divergence among populations of the Pacific oyster in north China (10). Linkage disequilibrium ( $\mathrm{LD}$, measured as $r^{2}$ ) decreased to 
152 half of its maximum value around $2.54-3.00 \mathrm{~kb}$ in the three populations (fig. S5),

153 which is substantially slower than the LD decay in the Pacific oyster $(\sim 0.1 \mathrm{~kb})(10)$.

154 These results provide unprecedented insights into the fine-scale population structure

155 of the estuarine oyster. The finding of exceptionally low sequence diversity, slow LD

156 decay and high population divergence in the estuarine oyster compared with the

157 Pacific oyster suggests that the two species experienced different evolutionary forces.

Historical glaciation, geological events and oceanographic forces shaping

diversity and distribution. Population structure of the estuarine oyster was largely concordant with the direction of ocean currents during the summer, where the northern or southern coastal currents are not crossed except over middle populations near the Yangtze River estuary (Fig. 2a and fig. S6). Nucleotide diversity $(\pi)$ was highest in middle populations $\left(3.56 \times 10^{-4}\right)$ and QD oysters $\left(3.59 \times 10^{-4}\right)$, likely because converging southern and northern currents bring increased genetic diversity to the middle populations (Fig. 3a). These findings indicate that ocean currents play important role in shaping and maintaining population structure of the estuarine oyster $(10,36)$. The exceptionally high divergence between SC and NC populations can be explained by the fact that currents from the south and north do not cross over and cannot facilitate gene flow. Freshwater discharge from Yangtze River is considered as a major barrier limiting gene flow between north and south populations of marine organisms $(8,37-40)$. For the estuarine oyster, however, populations on the north (NT) and south (SH) side of Yangtze River were clustered together as MC, indicating Yangtze River is not a barrier to gene flow for the estuarine oyster that thrives in low salinity water of estuaries.

To reconstruct the demographic history, Pairwise Sequentially Markovian Coalescent (PSMC) method were used to assess fluctuations in effective population size $(\mathrm{Ne})$ in response to Quaternary climatic change using genome data of two or three estuarine oysters from each of the three populations, as well as three previously sequenced 
glaciation events during the past million year as their $N e$ peaked at $\sim 0.90$ mya before the Mindel glaciation (MG, 0.68 0.80 mya) and then was substantially decreased over three periods of glaciation including MG, Riss glaciation (RG, 0.24 0.37 mya) and Würm glaciation (WG, 10,000 120,000 years ago). The Ne of both species reached their bottom during the last glaciation WG, but Pacific oyster populations rebounded early and to higher levels than the estuarine oyster, suggesting the last ice age had a greater impact on the estuarine oyster. Also, during the interglacial period before the last glaciation at $\sim 0.2$ mya, the SC and NC populations of the estuarine oyster started to diverge (Fig. 3b). We also estimated divergence time between SC and NC based on cytochrome oxidase I (COI) sequence data (29) using divergence time between $C$. gigas and $C$. angulata as reference $(39,41)$. The COI-based estimate placed the divergence time at 0.14 0.63 mya, which is close to the 0.2 mya estimated by PSMC with whole-genome data (Fig. 3b). The divergence time coincided with the formation land bridge between Taiwan and mainland China from 0.2 mya to 25,000 years ago by tectonic movement (42), creating a physical barrier to gene flow between southern and northern populations that is now maintained by ocean currents.

The nucleotide diversity of all three populations of estuarine oyster was 25 -fold lower than that of the Pacific oyster $\left(\pi_{-}\right.$. gigas $=9.27 \times 10^{-3}$, Fig. $\left.3 \mathrm{c}\right)$. Similarly, stickleback fishes adapted to freshwater areas exhibited lower $\pi$ and $N e$ values than counterparts dwelling in brackish areas (43). These findings suggest that climate change may affect species with different lifestyles differently and the estuarine oyster may be more sensitive to climate change than the Pacific oyster that inhabits more open waters of high salinity. Further, $\mathrm{Ne}$ curves of middle and northern populations split $\sim 90,000$ years ago, which corresponded to the great sea level fall at the sub-glaciation of WG when Bohai Sea dried up (44). The lower $\mathrm{Ne}$ and nucleotide diversity of northern population (Fig. 3a, b) support that a stronger bottleneck occurred in estuarine oysters of Bohai Sea. Relatively lower nucleotide diversity was also found in Bohai populations of the Pacific oyster, possibly due to the same sea level fall (10). The role of bottlenecks and geographic isolation resulting from historical glaciations and 
210 tectonic events in shaping phylogeography has been demonstrated in other molluscs,

211 such as the divergence between Atlantic coast and Gulf of Mexico populations of the 212 eastern oyster Crassostrea virginica (7). Our results provide putative divergence times 213 among estuarine oyster populations linked to historical events and suggest past 214 climate and tectonic movement played an important role in shaping genetic diversity 215 and population differentiation in the estuarine oyster.

216 To explore the effects of selection and genetic drift, we compared highly heterozygous

217 SNPs in northern and southern populations. The number of SNPs that are highly 218 heterozygous in NC only (14,373) were 1.89-fold higher than those highly 219 heterozygous in SC only (7,595) across 10 chromosomes (Fig. 3d). Both directional 220 selection and genetic bottleneck can decrease the number of heterozygous SNPs. The 221 finding that southern oysters had significantly higher ratio of genes with 222 non-synonymous SNPs $(35.67 \% \pm 0.35 \%)$ than the middle $(33.33 \% \pm 0.22 \%)$ and 223 northern $(31.82 \% \pm 0.29 \%)$ oysters $(p<0.01$, Fig. 3e), suggests that the southern oysters experienced stronger selection. Stronger selection, potentially from strong environmental disturbance, decreased the numbers of heterozygous SNPs in southern oysters. Thus, in addition to isolation and bottlenecks from historical glaciation, geological events and oceanographic forces, selection or local adaptation may also play an important role in shaping variation and phylogeography of the estuarine oyster.

230

Signatures of selection. Oysters inhabiting northern and southern estuaries experience significantly different environmental conditions. Southern habitats are characterized by high temperature and low salinity. Satellite remote sensing data from 2000 to 2017 indicated that the monthly average sea surface temperature (SST) of southern habitats was $10.35 \square$ higher than that of northern habitats (fig. S7). Salinity at the northern BZ site was $10.98 \%$ o higher than the southern TS site (3). Climate change may enhance the difference and cause salinity to increase at north habitats but decrease at south habitats $(45,46)$. Thus, temperature and salinity are the two most 
239 important environmental factors driving adaptive divergence between northern and

240 southern populations. With discontinuous distribution and limited gene flow, the

241 southern population became locally adapted and evolved higher tolerance to high

242 temperature and low salinity (3). We expect that some genomic regions were

243 subjected to selection and contributed to adaptation to higher temperature and lower

244 salinity conditions in the southern population.

245 To identify signatures of selection sweeps, we calculated the fixation index $\left(F_{\mathrm{ST}}\right)$ and 246 selection statistics (Tajima's $D$ ) between two population pairs (SC vs. NC and SC vs.

$247 \mathrm{MC}$ ) and identified $F_{\mathrm{ST}}$ outliers (top $1 \%, F_{\mathrm{ST} \_ \text {north vs. south }}>=0.693, F_{\mathrm{ST} \_ \text {middle vs. south }}>=$ 248 0.637). Only genomic regions surrounding selective peaks that overlapped between 249 the two population pairs and located at the valleys of Tajima's $D$ values in one of the 250 three populations were considered as under selection. A total of 24 selective regions 251 spanning 51 candidate genes (44 annotated) were identified along chromosomes 2, 3, 252 4, 6, 8 and 9 among three oyster populations (Fig. 4a, b and fig. S8-13, table S9). 253 Most of these candidate genes are involved in response to environmental disturbances 254 in salinity and temperature (47-54).

255 To assess transcriptomic response of the estuarine oyster to temperature and salinity 256 disturbances, we conducted RNA-seq analysis of oysters exposed to elevated 257 temperature (6 hours under $37 \square$ ) and high salinity (7 days under $60 \%$ ). Only genes 258 with 10 or more aligned reads in $>90 \%$ samples were used for subsequent analyses. 259 About $44.17 \%(10,279$ of 23,270$)$ and $11.7 \%(2,757$ of 23,650$)$ of genes were 260 differentially expressed under high salinity and temperature stresses, respectively, 261 with 1,088 genes responsive to both stressors (fig. S14). For the 51 candidate genes 262 from regions under selection, a total of 29 genes were expressed, and 75.9\% (22 of 29) 263 and $58.6 \%$ (17 of 29) of which were differentially expressed under high salinity and 264 high temperature, respectively ( $p<0.05$, Fig. 4c), indicating most genes under 265 selection are involved in response to environmental disturbances in these two factors.

266 Thirteen genes responded to both thermal and salinity stresses, while only three genes 267 were not sensitive to the two stressors. Nine and four genes highly responsive to high 
268 salinity and high temperature, respectively. The finding that most of genes from

269 regions under selection are involved in response to temperature and salinity 270 disturbances suggest that these two environmental factors are important drivers of 271 adaptive evolution in the estuarine oyster.

272

273 Expansion of genes contributing to temperature and salinity adaptation. Among

274 the 24 regions under selection, we found two clusters of tandemly duplicated genes 275 belonging to solute carrier families, 10 copies of Slc23a2 and four copies of 276 monocarboxylate transporter 12 (Mct12, also known as Slc16a12) (fig. S13c), located 277 in the two regions of chromosome 9 with high divergence $\left(F_{\mathrm{ST}}=0.81\right.$ and 0.76$)$ 278 among the three oyster populations (fig. S13d). The genomic region spanning Slc23a2 279 gene families exhibited extremely low Tajima's $D$ values in northern oysters, while 280 these spanning Mct12 gene families had extremely low Tajima's $D$ values in southern 281 oysters (fig. S13e). These findings indicate that Slc23a2 and Mct12 genes were under 282 directional selection in northern and southern environments respectively, highlighting 283 the critical role of $S l c$ family genes in adaptation of marine species, as also reported in 284 porpoises and coral $(47,55-57)$, to salinity and temperature variations.

285 The ten copies of Slc23a2 family belong to three orthogroups, where two of them are 286 annotated as purine permease (a: OG0011985 and c: OG0000489) and the other is 287 annotated as uric acid transporter (OG0000633). Four copies of Mct12 gene family 288 belong to one orthogroup annotated as purine efflux pump (OG0000571). All these 289 orthogroups were extensively expanded in the estuarine oyster, as well as in other two 290 other oyster species (Crassostrea virginica and Crassostrea hongkongensis) that occur 291 in warm and low-salinity estuarine environments, but not so in C. gigas that inhabit 292 cool, open and high-salinity waters (fig. S15). Three copies of Slc23a2 (Slc23a2_al, 293 Slc23a2_a2 and Slc23a2_c2) in two expanded orthogroups responded to both 294 temperature and salinity challenges, while three copies of Mct12 (Mct12_1, Mct12_2 295 and Mct12_3) responded to salinity challenge (Fig. 4c). The expansion of these 296 environment-responsive genes may enhance transcriptional complexity or plasticity 
297

298

299

300

301

302

303

304

305

306

307

308

309

310

311

312

313

314

315

316

317

318

319

320

321

322

323

324

325

and play an important role in adaption to diverse temperature and salinity conditions in the estuarine oyster and two other species inhabiting similar environments. This finding provides further evidence that gene duplication is critical for stress adaptation, as previously demonstrated for the expansion of heat shock protein and inhibitor of apoptosis gene families in the Pacific oyster and other marine bivalves $(22,31,58)$.

\section{Stronger selection in upstream regulatory regions of environment-responsive} genes with high plasticity. To understand the interaction between divergence and plasticity, we examined divergence in different genomic regions (genic, upstream and downstream) with $F_{\mathrm{ST}}$ and transcriptional plasticity of the 29 genes from regions under selection in response to environmental translocations. $F_{1}$ progeny produced from northern and southern populations were acclimatized at both northern and southern habitats for three months before transcriptional analysis. Fourteen of the 29 genes exhibited higher plasticity (HP) where they showed significantly differentiated expression when translocated to nonnative environments in both oyster populations ( $p$ $<0.05$ ), while other genes had lower plasticity (LP) and their expression didn't strongly respond to environmental translocation/change (Fig. 5a). The transcriptional plasticity of the HP genes was $19.15 \%$ higher than that of LP genes $(p=0.0255$, fig. S16).

For the whole genome, genic regions showed significantly higher $F_{\mathrm{ST}}$ values (mean $\left.F_{\mathrm{ST} \_ \text {genic }}=0.163\right)$ than that of intergenic regions $\left(F_{\mathrm{ST} \_ \text {intergenic }}=0.138, p<0.001\right.$, Wilcoxon signed-rank tests, Fig. 5b), indicating stronger selection in genic regions driving differentiation between northern and southern populations. For all 29 genes from regions under selection, both genic $\left(F_{\mathrm{ST}}=0.7745\right)$ and intergenic $\left(F_{\mathrm{ST}}=0.7585\right)$ regions showed strong selection signals or divergence compared with all genes in the genome, and the difference between genic and intergenic regions was not significant $(p>0.05)$. For both genic and intergenic regions, genes with high plasticity showed significantly $(p=0.0230)$ higher divergence $\left(F_{\mathrm{ST}}=0.7816\right)$ between northern and southern oysters than LP genes $\left(F_{\mathrm{ST}}=0.7274\right)$ (Fig. $\left.5 b\right)$. Specifically, the upstream 
326 intergenic regions of HP genes exhibited significantly $(p=0.01512)$ higher

327 divergence $\left(F_{\mathrm{ST}}=0.7958\right)$ between northern and southern populations than that of LP

328 genes $\left(F_{\mathrm{ST}}=0.7402\right)$, while there was no significant difference between the two type

329 of genes for genic and downstream intergenic regions ( $p>0.05$, Fig. 5c). Further, LP

330 genes showed higher divergence in genic regions. While selection preferentially

331 targeted genic regions at genome level and for LP genes, HP genes showing high

332 plasticity exhibited stronger selection in upstream intergenic regions, where critical

333 regulatory elements such as promoter and enhancers reside, potentially regulate gene

334 expression and enhance transcriptional plasticity (19). These results suggest that

335 selection and divergence in upstream regulatory regions may enhance phenotypic

336 plasticity that contributes to the survival and adaption of organisms facing

337 environmental disturbances. The mechanism of selection favoring plasticity is not

338 clear at this time. Directional selection tends to reduce genetic variation and facilitate

339 local adaptation. Selection acting on regulatory elements may create divergence and

340 transcriptional complexity that enhance phenotypic plasticity $(18,19)$ at species level.

341 Directional selection acting on duplicated genes can potentially direct duplicated

342 member genes to express under different conditions and therefore enhance overall

343 phenotypic plasticity. It is also possible that balancing selection retains diversity in

344 regulatory elements and increase plasticity. It has been suggested that balancing

345 selection is pervasive in oysters and important for their evolution (59).

346 In conclusion, analysis of the estuarine oyster genome revealed exceptionally low

347 genetic diversity and fine-scale population structures, which were shaped and

348 maintained by climate history, geological events and oceanographic forces.

349 Integration of genomic and transcriptomic analyses revealed genes in regions under

350 selection are mostly involved in environmental response including the expanded

351 solute carrier gene families that are important for environmental adaptation.

352 Expansion and selection in upstream regulatory regions of environment-responsive

353 genes may enhance transcriptional complexity and phenotypic plasticity that are

354 critical for the adaptation to rapidly changing environments. 


\section{Materials and Methods}

356 Genome sequencing. A wild adult estuarine oyster Crassostrea ariakensis was 357 obtained from Binzhou, Bohai Sea, northern China. Four tissue samples (gill, mantle, 358 adductor muscle and labial palp) were collected and flash-frozen in liquid nitrogen.

359 Genomic DNA was extracted from the adductor muscle and used to construct libraries for Oxford Nanopore Technologies' long-read sequencing. In addition, libraries with insert size of $\sim 350$ bp were prepared and sequenced using Illumina HiSeq 4000 platform.

363

Genome size and heterozygosity. Trimmed Illumina short reads were used to generate the k-mer copy number $(\mathrm{KCN})$ distribution. We selected 21-mer for $\mathrm{KCN}$ distribution which showed two distinct peaks (fig. S1). The first peak $(\mathrm{KCN}=45)$ represents heterozygous single copy k-mers while the second peak $(\mathrm{KCN}=90)$ represents homozygous single copy k-mers in the genome. Genome size was estimated by the formula $\mathrm{G}=\mathrm{K} \_$num/peak depth.

371 Contig-level assembly using long-reads. Nanopore long reads, with a read N50 of 37233,230 and a mean read length of $23,240 \mathrm{bp}$, were used for initial genome assembly. 373 Error correction of clean data was conducted using Canu (60) v1.5, and then were 374 assembled using Canu, WTDBG2 (61) and SMARTdenovo tools. Quickmerge (62) 375 v0.2.2 was used to join the three assemblies, and the assembly was corrected for 3 376 cycles using long reads by Racon (63) and for 3 cycles using Illumina reads by Pilon 377 (64) v1.22 with default parameters. The initial assembly of the estuarine oyster 378 genome was $613,892,480$ bp in length with a contig N50 of $6,967,240 \mathrm{bp}$.

380 Chromosome-level assembly with Hi-C. The same genomic DNA extracted from the adductor muscle was used to construct Hi-C library for with the Illumina HiSeq 4000 platform. Contigs and scaffolds which were then sorted and oriented into super scaffolds using LACHESIS (65) with the following parameters: cluster_min_re_sites 
$384=47, \quad$ cluster_max_link_density $=2, \quad$ cluster_noninformative_ratio $=2$,

385 order_min_n_res_in_trun $=40$, order_min_n_res_in_shreds $=41$.

387 Genome evaluation. Hi-C contact heatmap of the number of Hi-C links between 100

$388 \mathrm{~kb}$ windows on the pseudochromosomes was used to assess the accuracy of the Hi-C

389 assembly. Benchmarking Universal Single-Copy Orthologs (BUSCO) v3.0.2 with 978

390 conserved genes were used to assess the completeness and accuracy of gene coverage.

391 The completeness and accuracy of the assembly was also evaluated by mapping rate

392 of RNA-seq reads (see Protein coding gene annotation) and Illumina genomic reads

393 with Burrows-Wheeler Aligner (66) v.0.7.8.

394

395 Repeat annotation. Transposable elements (TEs) were identified and classified using 396 homology and de novo-based approaches. RepeatScout and LTR_FINDER were used 397 to construct de novo repeat libraries. The de novo-based libraries were further 398 classified with PASTEClassifier (67) to obtain a consensus library, and combined with 399 the repeat library of Repbase data. RepeatMasker (68) v4.0.5 was used to identify TEs 400 in the estuarine oyster genome with the combined library.

401

402 Protein-coding gene annotation. Protein-coding genes were identified with three 403 methods: de novo prediction, homology-based prediction and RNA-seq-based 404 prediction. For de novo prediction, five ab initio gene prediction programs, Genscan 405 (69) v1.0, Augustus (70) v2.4, GlimmerHMM (71) v3.0.4, GeneID (72) v1.4 and $406 \operatorname{SNAP}(73)$, were used to predict genes in the repeat-masked genome. For 407 homolog-based prediction, protein sequences from 10 well-annotated species, 408 including Homo sapiens, Danio rerio, Aplysia californica, Strongylocentrotus 409 purpuratus, C. gigas, C. virginica, Biomphalaria glabrata, Lingula anatina, Octopus 410 bimaculoides and Mizuhopecten yessoensis, were downloaded from GenBank and 411 aligned to the repeat-masked estuarine oyster genome using tblastn (74) with E-value $412 \leq 1 \mathrm{E}-05$. We used GeMoMa (75) v1.3.1 to predict gene models based on alignment 
413 sequences. For RNA-seq-based prediction, RNA-seq data generated from four tissues

414 (gill, mantle, adductor muscle and labial palp) using ONT long-read sequencing

415 platform were filtered to remove adaptors and then trimmed to remove low-quality

416 bases. Clean reads were aligned to reference genome using TopHat2 (76) and then

417 assembled using Trinity (77). Full transcriptome-based genome annotation was

418 predicted using PASA (78) v2.2.2. Finally, EVidenceModeler (79) (EVM) v1.1.1 was

419 used to generate a weighted and non-redundant gene set by integrating all gene

420 models predicted by the three methods.

421 Homologous sequences in the genome were identified with genBlastA (80) v1.0.4

422 using the integrated gene set, and GeneWise (81) was used to identify pseudogenes.

423 Transfer RNAs (tRNAs) were defined using tRNAscan-SE (82) v1.3.1 software with

424 eukaryote default parameters. MicroRNA and rRNA were identified with Infernal

425 BLASTN (83) against the Rfam (84) database v12.0.

426 Functional annotation of protein-coding genes was conducted by aligning them to the

427 NCBI non-redundant protein (85) (NR), SwissProt (86), KOG (87) and TrEMBL (86)

428 databases using BLAST (83) v2.2.31 with a maximal e-value of 1e-05. Domains were

429 identified by searching against Pfam (88) database using HMMER (89) v3.0. Genes

430 were mapped to Gene Ontology (GO) terms and KEGG pathways to identify their

431 best functional classification.

432

433 Whole-genome resequencing and mapping. We collected 264 wild estuarine oysters

434 from 11 estuaries (Fig. 3a), covering most of its distribution range (26, 27). Genomic

435 DNA was isolated from gill tissue with standard phenol-chloroform extraction and

436 used to construct paired-end libraries with an insert size of $\sim 350 \mathrm{bp}$ according to the

437 manufacturer's instructions (Illumina Inc., San Diego, CA, USA) for sequencing on

438 the Illumina HiSeq X Ten Sequencer. We obtained $\sim 14.42 \mathrm{~Gb}$ of clean data for each

439 sample, giving an average depth of 19.9X coverage (15-28X) (table S8). The 150-bp

440 paired-end reads were mapped to the estuarine oyster reference genome

441 (PRJNA715058) with the BWA (66) using default parameters (bwa mem -M -t 10 -T 
442 20). Mapping data were then converted into the BAM format and sorted by SAMtools

443 v.1.3.1 (90) to remove duplicate reads. Read pairs with the highest mapping quality

444 were retained if multiple pairs have identical external coordinates.

445

446 Variant calling and annotation. The Genome Analysis Toolkit (GATK) v.3.7 (91)

447 module HaplotypeCaller was used to obtain high-quality variation calling of each

448 sample. SNPs were further filtered with parameters 'QD<2.0 || FS>60.0 || MQ<40.0'.

449 Similarly, indels were called and filtered using parameters 'QD<2.0 \| FS>60.0'.

450 Filtered SNPs were annotated by SnpEff (92) and then classified into regions of exon,

451 intron, splicing site, and upstream and downstream intergenic regions, and as

452 heterozygous or homozygous variants. To characterize the types of variants in

453 northern and southern oysters, Plink (93) was used to filter raw SNPs using

454 parameters of MAF $>0.05$ and Int $>0.8$. The retained SNPs were singly classified as

455 highly heterozygous in a population if more than half individuals were heterozygous

456 in that population but not in the other population. Variations in exons were further

457 categorized as synonymous or non-synonymous SNPs. Two-sided two-sample

458 Wilcoxon signed-rank tests were conducted to test whether the ratios of genes with

459 nonsynonymous mutations were different between northern and southern populations,

460 using the function wilcoxsign-test in R package "coin".

461

462 Population analysis. Population structure was inferred using ADMIXTURE v.1.23

463 (94) with default settings. The number of assumed genetic clusters $K$ ranged from 2 to

4645 , and the optimum $K$ was assessed with cross-validation errors. The individual-based

465 neighbor-joining (NJ) phylogenetic tree was constructed using MEGA (95) under

466 Kimura 2-Parameter model with 1000 bootstraps, and visualized using FigTree. PCA

467 was performed with whole-genome SNPs of all 264 individuals using Eigensoft (96).

468 To evaluate LD decay, parameter $r^{2}$ between any two loci was calculated within each

469 chromosome using Plink v.1.07 (93) with the command -ld-window-r2 0 -ld-window

47099999 -ld-window-kb 500. The average $r^{2}$ values were calculated for each length of 
471 distance and the genome-wide LD was averaged across all chromosomes. The LD

472 decay was plotted against the length of distance. Popgenome R package (97) was used

473 to calculate Tajima's $D$, global $F_{\mathrm{ST}}$ and nucleotide diversity $(p)$ using a 100 -kb sliding

474 window with the step size of $10-\mathrm{kb}$.

475

476 Demographic history. We implemented PSMC (98) to estimate dynamics of effective

477 population size $(\mathrm{Ne})$ and possible divergence time over the past several million years.

478 A total of eight estuarine oysters from northern $(n=3)$, middle $(n=2)$ and southern $(n$

$479=3)$ populations and three Pacific oysters (10) with high sequencing depth $(20-28 \times)$

480 were used. To minimize the probability of false positives, sequencing depth of SNPs

481 was filtered with parameters: MinDepth $=$ average depth $/ 3$, MaxDepth $=$ average

482 depth $\times 2$. The PSMC parameters were set as: $-\mathrm{N} 25$-t15 -r5 -p ' $4+25 * 2+4+6$ ' to

483 estimate historical $N e$. The estimated generation time $(g)$ was set as 1 for both species,

484 while mutation rates $(\mu)$ were calculated, following the formula $\mathrm{T}_{\text {divergence }}=\mathrm{Ks} / 2 \mu$, as

$4850.3 \times 10^{-8}$ and $0.2 \times 10^{-8}$ for estuarine and Pacific oysters, respectively.

487 Selection signature detection. To identify selection sweeps potentially contributing 488 to adaptation to southern environments, we calculated fixation $\left(F_{\mathrm{ST}}\right)$ and selection 489 statistics (Tajima's $D$ ) between two pairs of populations, north vs. south and middle vs. 490 south, in a 100-kb sliding window with a step size of 10-kb. Genomic regions 491 showing strong selection signals were defined as: 1$)$ regions with top $1 \% F_{\mathrm{ST}}$ values 492 that overlapped in both pair comparisons; and 2) regions located at valleys of Tajima's $493 D$ values distribution along the chromosome in one of the three populations.

495 Response to temperature and salinity disturbance. To investigate responses of 496 estuarine oysters to challenges of elevated temperature and high salinity, we collected 497 wild oysters and exposed them to different temperatures (20 $\square$ and $37 \square$ for 6 hours) 498 and salinities (20 and 60 for 7 days), respectively. Gills from five oysters were 499 individually sampled and flash-frozen in liquid nitrogen for subsequent transcriptomic 
500 analysis.

501

502 Reciprocal transplantation experiments. To explore the plasticity of candidate 503 genes from regions under selection, we measured transcriptional changes in oysters 504 subjected to native and non-native environments after reciprocal transplantation (3). 505 Briefly, wild oysters derived from northern (Binzhou: BZ, Bohai Sea) and southern 506 (Taishan: TS, East China Sea) environments were collected and used to produce $\mathrm{F}_{1}$ 507 progeny. For each population, 40 males and 40 females were selected as parents. Eggs 508 were pooled and then divided into 40 beakers, each fertilized with sperm from one of 509 the 40 males to create all possible matings. Zygotes fathered by every eight males 510 were combined and cultured as one group, and the five groups were reared in separate 511 tanks in the hatchery and nursery to juvenile stage. Two-month-old $F_{1}$ juveniles from 512 each of the two populations were outplanted at two source habitats to assess their 513 responses to reciprocal transplantation or environmental change. After three months 514 of acclimation at northern and southern environments, gills of five oysters were 515 sampled from each of population at both habitats in situ and flash-frozen in liquid 516 nitrogen for subsequent transcriptomic analysis.

517

518 Transcriptomic analysis. Total RNA was isolated from gills sampled from laboratory 519 challenge experiment (high temperature and high salinity) and field reciprocal 520 transplantation experiment, using the RNAprep Pure Tissue Kit (Tiangen) following 521 the manufacturer's protocol. The RNA integrity and concentration were examined 522 with 1.2\% agarose gel electrophoresis and Nanodrop 2000 spectrophotometer, 523 respectively. DNA contamination was removed with DNAse I treatment. RNA 524 integrity was further assessed using an Agilent Bioanalyzer 2100 and the RNA Nano 5256000 Assay Kit. One $\mu$ g RNA per sample was used to construct sequencing libraries 526 using NEBNext Ultra ${ }^{\mathrm{TM}}$ RNA Library Prep Kit for 150-bp paired-end sequencing on 527 an Illumina HiSeq 4000 platform. Clean data were obtained by removing reads 528 containing adapters, reads containing ploy- $\mathrm{N}$ and low-quality reads. TopHat2 was 
529 used to map clean reads to the estuarine oyster genome. StringTie v2.0 was used for

530 read assembly. Only reads with perfect match or one mismatch were further analyzed

531 and annotated. Gene expression levels were estimated by fragments per kilobase of 532 transcript per million fragments mapped (FPKM). We used DESeq2 to determine 533 differentially expressed genes (DEGs) between different populations or treatments.

534 Genes with an adjusted $p$-value $<0.01$ using the Benjamin and Hochberg's approach

535 were accepted as DEGs. For genes from regions under selection, those significantly 536 differentially expressed between northern and southern habitats in both oyster 537 populations were defined as high plasticity genes (HP), while others were defined as 538 low plasticity genes (LP). A hierarchical cluster analysis was performed to show 539 differential expression of these genes, using the pheatmap package in $\mathrm{R}$ software.

540

\section{Supplementary materials}

542 Supplementary material for this article is available at the end of this file.

543

544 References and Notes

545 1. O. Hoegh-Guldberg, J. F. Bruno, The impact of climate change on the world's marine $546 \quad$ ecosystems. Science 328, 1523-1528 (2010).

547 2. C. Chou, J. C. H. Chiang, C.-W. Lan, C.-H. Chung, Y.-C. Liao, C.-J. Lee, Increase in 548 the range between wet and dry season precipitation. Nature Geoscience 6, 263-267 $549 \quad$ (2013).

550 3. A. Li, C. Wang, W. Wang, Z. Zhang, M. Liu, Z. She, Z. Jia, G. Zhang, L. Li, Molecular 551 and Fitness Data Reveal Local Adaptation of Southern and Northern Estuarine $552 \quad$ Oysters (Crassostrea ariakensis). Frontiers in Marine Science 7, (2020).

553 4. R. F. Schneider, A. Meyer, How plasticity, genetic assimilation and cryptic genetic 
555 5. E. Crispo, The Baldwin effect and genetic assimilation: revisiting two mechanisms of evolutionary change mediated by phenotypic plasticity. Evolution 61, 2469-2479 (2007).

6. D. W. Pfennig, M. A. Wund, E. C. Snell-Rood, T. Cruickshank, C. D. Schlichting, A. P. Moczek, Phenotypic plasticity's impacts on diversification and speciation. Trends in ecology \& evolution 25, 459-467 (2010).

7. N. E. Buroker, W. K. Hershberger, K. K. Chew, Population Genetics of the Family Biology 54, 171-184 (1979).

8. G. Ni, E. Kern, Y. W. Dong, Q. Li, J. K. Park, More than meets the eye: The barrier effect of the Yangtze River outflow. Molecular ecology 26, 4591-4602 (2017).

9. Y. Xiao, D. Ma, M. Dai, Q. Liu, Z. Xiao, J. Li, J. Liu, S. Xu, Q. Cheng, The impact of Yangtze River discharge on the genetic structure of a population of the rock bream, Oplegnathus fasciatus. Marine Biology Research 12, 426-434 (2016). plasticity shape adaptive potential of the Pacific oyster. Nat Ecol Evo/2, 1751-1760 (2018).

574 11. C. D. Kenkel, M. V. Matz, Gene expression plasticity as a mechanism of coral 575 adaptation to a variable environment. Nature Ecology \& Evolution 1, 0014 (2016). 
576 12. T. Armarego-Marriott, Climatic selection and gene expression plasticity. Nature

577

578

579

580

581

582

583

584

585

586

587

588

589

590

591

592

593

594

595

596

597

Climate Change 11, 4-4 (2021).

13. C. A. Vargas, N. A. Lagos, M. A. Lardies, C. Duarte, P. H. Manriquez, V. M. Aguilera, B. Broitman, S. Widdicombe, S. Dupont, Species-specific responses to ocean acidification should account for local adaptation and adaptive plasticity. Nature Ecology \& Evolution 1, 84 (2017).

14. L. Y. Yampolsky, T. M. Schaer, D. Ebert, Adaptive phenotypic plasticity and local adaptation for temperature tolerance in freshwater zooplankton. Proceedings. Biological sciences / The Royal Society 281, 20132744 (2014).

15. J. Sandoval-Castillo, K. Gates, C. J. Brauer, S. Smith, L. Bernatchez, L. B. Beheregaray, Adaptation of plasticity to projected maximum temperatures and across climatically defined bioregions. Proceedings of the National Academy of Sciences 117, $17112-17121(2020)$

16. D. W. Whitman, A. A. Agrawal, What is phenotypic plasticity and why is it important? (Phenotypic Plasticity of Insects, Science Publishers, 2009).

17. L. E. Eierman, M. P. Hare, Reef-Specific Patterns of Gene Expression Plasticity in Eastern Oysters (Crassostrea virginica). The Journal of heredity 107, 90-100 (2016).

18. M. Kelly, Adaptation to climate change through genetic accommodation and assimilation of plastic phenotypes. Philosophical Transactions of the Royal Society B: Biological Sciences 374, 20180176 (2019).

19. V. Grishkevich, I. Yanai, The genomic determinants of genotype $\mathrm{x}$ environment interactions in gene expression. Trends in genetics : T/G 29, 479-487 (2013). 
598 20. A. Li, L. Li, K. Song, W. Wang, G. Zhang, Temperature, energy metabolism, and 599 adaptive divergence in two oyster subspecies. Ecology and evolution 7, 6151-6162 $600 \quad$ (2017).

601 21. P.-A. Gagnaire, J.-B. Lamy, F. Cornette, S. Heurtebise, L. Degremont, E. Flahauw, P. 602 Boudry, N. Bierne, S. Lape'gue, Analysis of Genome-Wide Differentiation between 603 Native and Introduced Populations of the Cupped Oysters Crassostrea gigas and 605 22. G. Zhang, X. Fang, X. Guo, L. Li, R. Luo, F. Xu, P. Yang, L. Zhang, X. Wang, H. Qi, Z. 606 Xiong, H. Que, Y. Xie, P. W. Holland, J. Paps, Y. Zhu, F. Wu, Y. Chen, J. Wang, C.

23. A. Li, L. Li, W. Wang, G. Zhang, Evolutionary trade-offs between baseline and plastic gene expression in two congeneric oyster species. Biology Letters 15, 20190202 618 (2019).

619 24. M. W. Beck, R. D. Brumbaugh, L. Airoldi, A. Carranza, L. D. Coen, C. Crawford, O. 
620

621

622

623

624

625

626

627

628

629

630

631

632

633

634

635

636

637

638

639

640

641

Defeo, G. J. Edgar, B. Hancock, M. C. Kay, H. S. Lenihan, M. W. Luckenbach, C. L. Toropova, G. Zhang, X. Guo, Oyster Reefs at Risk and Recommendations for Conservation, Restoration, and Management. BioScience61, 107-116 (2011).

25. X. Guo, S. E. Ford, Infectious diseases of marine molluscs and host responses as revealed by genomic tools. Philosophical transactions of the Royal Society of London. Series B, Biological sciences 371, (2016).

26. H. Wang, X. Guo, G. Zhang, F. Zhang, Classification of jinjiang oysters Crassostrea rivularis (Gould, 1861) from China, based on morphology and phylogenetic analysis. Aquaculture 242, 137-155 (2004).

27. M. F. Zhou, S. K. Allen, A review of published work on Crassostrea ariakensis. Journal of Shellfish Research 22, 1-20 (2003).

28. H. Wang, L. Qian, G. Zhang, X. Liu, A. Wang, Y. Shi, N. Jiao, X. Guo, Distribution of Crassostrea ariakensis in China. Journal of Shellfish Research 25, 789-790 (2006).

29. C. M. Bai, L. S. Xin, U. Rosani, B. Wu, Q. C. Wang, X. K. Duan, Z. H. Liu, C. M. Wang, Chromosomal-level assembly of the blood clam, Scapharca (Anadara) broughtonii, using long sequence reads and Hi-C. GigaScience 8, (2019).

30. J. Peng, Q. Li, L. Xu, P. Wei, P. He, X. Zhang, L. Zhang, J. Guan, X. Zhang, Y. Lin, J. Gui, X. Chen, Chromosome-level analysis of the Crassostrea hongkongensis genome reveals extensive duplication of immune-related genes in bivalves. Molecular ecology resources 20, 980-994 (2020).

31. H. Song, X. Guo, L. Sun, Q. Wang, F. Han, H. Wang, G. A. Wray, P. Davidson, Q. Wang, Z. Hu, C. Zhou, Z. Yu, M. Yang, J. Feng, P. Shi, Y. Zhou, L. Zhang, T. Zhang, 
642

643

644 32. C. Penaloza, A. Gutierrez, L. Eory, S. Wang, X. Guo, A. Archibald, T. Bean, R. 645 Houston, A chromosome-level genome assembly for the Pacific oyster Crassostrea

646

647

648

649

650

651

652

653

654

655

656

657

658

659

660

661

662

663

The hard clam genome reveals massive expansion and diversification of inhibitors of apoptosis in Bivalvia. BMC Bio/19, 15 (2021).

gigas. GigaScience 10, (2021).

33. J. Xiao, J. F. Cordes, H. Wang, X. Guo, K. S. Reece, Population genetics of Crassostrea ariakensis in Asia inferred from microsatellite markers. Marine Biology $157,1767-1781$ (2010).

34. W.-J. Kim, S. T. Dammannagoda, H. Jung, I. S. Baek, H. S. Yoon, S. D. Choi, Mitochondrial DNA sequence analysis from multiple gene fragments reveals genetic heterogeneity of Crassostrea ariakensis in East Asia. Genes Genom. 36, 611-624 (2014).

35. X. Liu, L. Li, A. Li, Y. Li, W. Wang, G. Zhang, Transcriptome and Gene Coexpression Network Analyses of Two Wild Populations Provides Insight into the High-Salinity Adaptation Mechanisms of Crassostrea ariakensis. Marine biotechnology 21, 596-612 (2019).

36. C. Li, M. Olave, Y. Hou, G. Qin, R. F. Schneider, Z. Gao, X. Tu, X. Wang, F. Qi, A. Nater, A. F. Kautt, S. Wan, Y. Zhang, Y. Liu, H. Zhang, B. Zhang, H. Zhang, M. Qu, S. Liu, Z. Chen, J. Zhong, H. Zhang, L. Meng, K. Wang, J. Yin, L. Huang, B. Venkatesh, A. Meyer, X. Lu, Q. Lin, Genome sequences reveal global dispersal routes and suggest convergent genetic adaptations in seahorse evolution. Nature communications 12, 1094 (2021). 
664 37. Y. Dong, H. Wang, G. Han, C. Ke, X. Zhan, T. Nakano, G. A. Williams, The Impact of

665

666

667

668

669

670

671

672

673

674

675

676

677

678

679

680

681

682

683

684

685

Yangtze River Discharge, Ocean Currents and Historical Events on the Biogeographic Pattern of Cellana toreuma along the China Coast. PloS ONE7, e36178 (2012).

38. G. Ni, Q. Li, L. Kong, X. D. Zheng, Phylogeography of bivalve Cyclina sinensis. testing the historical glaciations and Changjiang River outflow hypotheses in northwestern Pacific. PLOS ONE7, e49487 (2012).

39. H. Wang, L. Qian, X. Liu, G. Zhang, X. Guo, Classification of a common cupped oyster from southern China. Journal of Shellfish Research 29, 857-866 (2010).

40. H. Wang, G. Zhang, X. Liu, X. Guo, Classification of Common Oysters from North China. Journal of Shellfish Research 27, 495-503 (2008).

41. J. Ren, X. Liu, F. Jiang, X. Guo, B. Liu, Unusual conservation of mitochondrial gene order in Crassostrea oysters: evidence for recent speciation in Asia. BMC evolutionary biology 10, 394 (2010).

42. M. Kimura, Paleogeography of the Ryukyu Islands. Tropics 10, 5-24 (2000).

43. J. A. M. Raeymaekers, A. Chaturvedi, P. I. Hablutzel, I. Verdonck, B. Hellemans, G. E. Maes, L. De Meester, F. A. M. Volckaert, Adaptive and non-adaptive divergence in a common landscape. Nature communications 8, 267 (2017).

44. Y. Qin, Y. Zhao, S. Zhao, Geology of the Bohai Sea (Science Press, Beijing, 1985).

45. N. Skliris, R. Marsh, S. A. Josey, S. A. Good, C. Liu, R. P. Allan, Salinity changes in the World Ocean since 1950 in relation to changing surface freshwater fluxes. Climate Dynamics 43, 709-736 (2014).

46. M. M. Mekonnen, A. Y. Hoekstra, Four billion people facing severe water scarcity. 
686

687

688

689

690

691

692

693

694

695

696

697

698

699

700

701

702

703

704

705

706

707

Science Advances 2, e1500323 (2016).

47. X. Zhou, X. Guang, D. Sun, S. Xu, M. Li, I. Seim, W. Jie, L. Yang, Q. Zhu, J. Xu, Q. Gao, A. Kaya, Q. Dou, B. Chen, W. Ren, S. Li, K. Zhou, V. N. Gladyshev, R. Nielsen, X. Fang, G. Yang, Population genomics of finless porpoises reveal an incipient cetacean species adapted to freshwater. Nature communications 9, 1276 (2018).

48. C. A. Ribeiro, F. Balestro, V. Grando, M. Wajner, Isovaleric acid reduces $\mathrm{Na}+$, K+-ATPase activity in synaptic membranes from cerebral cortex of young rats. Cellular and molecular neurobiology 27, 529-540 (2007).

49. Y. Huang, J. Niwa, G. Sobue, G. E. Breitwieser, Calcium-sensing receptor ubiquitination and degradation mediated by the E3 ubiquitin ligase dorfin. The Journal of biological chemistry 281, 11610-11617 (2006).

50. H. Vienken, N. Mabrouki, K. Grabau, R. F. Claas, A. Rudowski, N. Schomel, J. Pfeilschifter, D. Lutjohann, G. van Echten-Deckert, D. Meyer Zu Heringdorf, Characterization of cholesterol homeostasis in sphingosine-1-phosphate lyase-deficient fibroblasts reveals a Niemann-Pick disease type C-like phenotype with enhanced lysosomal $\mathrm{Ca}(2+)$ storage. Scientific reports 7, 43575 (2017).

51. M. Pagano, M. A. Clynes, N. Masada, A. Ciruela, L.-J. Ayling, S. Wachten, D. M. F. Cooper, Insights into the residence in lipid rafts of adenylyl cyclase AC8 and its regulation by capacitative calcium entry. Am J Physiol Cell Physio/ 296, C607-C619 (2009).

52. E. J. Weinman, W. P. Dubinsky, S. Shenolikar, Reconstitution of cAMP-Dependent Protein Kinase Regulated Renal $\mathrm{Na}^{+}-\mathrm{H}^{+}$Exchanger. J. Membr. Biol. 101, 11-18 
53. M. C. Fonteles, R. N. Greenberg, H. S. A. Monteiro, M. G. Currie, L. R. Forte, Natriuretic and kaliuretic activities of guanylin and uroguanylin in the isolated perfused rat kidney. The American Journal of PhysiologyF191-F197, (1998).

54. D. B. Stern, C. E. Lee, Evolutionary origins of genomic adaptations in an invasive copepod. Nat Ecol Evol4, 1084-1094 (2020).

55. A. M. Barrio, S. Lamichhaney, G. Fan, N. Rafati, M. Pettersson, H. Zhang, J. Dainat, D. genetic basis for ecological adaptation of the Atlantic herring revealed by genome sequencing. eLife 5, e12081 (2016).

56. C. D. Kenkel, E. Meyer, M. V. Matz, Gene expression under chronic heat stress in environments. Molecular ecology22, 4322-4334 (2013). families have a remarkably long evolutionary history with the majority of the human families present before divergence of Bilaterian species. Mol Biol Evol28, 1531-1541 (2011). oysters with insights on adaptation. Fish \& shellfish immunology 46, 107-119 (2015). 
60. S. Koren, B. P. Walenz, K. Berlin, J. R. Miller, N. H. Bergman, A. M. Phillippy, Canu: scalable and accurate long-read assembly via adaptive k-mer weighting and repeat separation. Genome research 27, 722-736 (2017).

61. V. Jayakumar, Y. Sakakibara, Comprehensive evaluation of non-hybrid genome assembly tools for third-generation PacBio long-read sequence data. Briefings in bioinformatics 20, 866-876 (2019).

62. M. Chakraborty, J. G. Baldwin-Brown, A. D. Long, J. J. Emerson, Contiguous and accurate de novo assembly of metazoan genomes with modest long read coverage. Nucleic acids research 44, e147 (2016).

63. R. Vaser, I. Sovic, N. Nagarajan, M. Sikic, Fast and accurate de novo genome assembly from long uncorrected reads. Genome research 27, 737-746 (2017).

64. B. J. Walker, T. Abeel, T. Shea, M. Priest, A. Abouelliel, S. Sakthikumar, C. A. Cuomo,

65. J. N. Burton, A. Adey, R. P. Patwardhan, R. Qiu, J. O. Kitzman, J. Shendure, Chromosome-scale scaffolding of de novo genome assemblies based on chromatin interactions. Nature biotechnology 31, 1119-1125 (2013). transform. Bioinformatics 25, 1754-1760 (2009). 

ONE 9, e91929 (2014).

68. M. Tarailo-Graovac, N. Chen, Using RepeatMasker to identify repetitive elements in genomic sequences. Curr Protoc Bioinformatics Chapter 4, Unit 410 (2009).

69. C. Burge, S. Karlin, Prediction of complete gene structures in human genomic DNA. Journal of molecular biology 268, 78-94 (1997).

70. M. Stanke, S. Waack, Gene prediction with a hidden Markov model and a new intron submodel. Bioinformatics 19 Suppl 2, ii215-225 (2003).

71. W. H. Majoros, M. Pertea, S. L. Salzberg, TigrScan and GlimmerHMM: two open source ab initio eukaryotic gene-finders. Bioinformatics 20, 2878-2879 (2004). Bioinformatics 64, e56 (2018).

73. I. Korf, Gene finding in novel genomes. Bmc Bioinformatics 5, 59 (2004).

74. S. F. Altschul, T. L. Madden, A. A. Schäffer, J. Zhang, Z. Zhang, W. Miller, D. J. Lipman, Gapped BLAST and PSI-BLAST: a new generation of protein database search programs. Nucleic acids research 25, 3389-3402 (1997). Bioinformatics 19, 189 (2018). 
774 77. M. G. Grabherr, B. J. Haas, M. Yassour, J. Z. Levin, D. A. Thompson, I. Amit, X.

775

776

777

778

779

780

781

782

783

784

785

786

787

788

789

790

791

792

793

794

795
Adiconis, L. Fan, R. Raychowdhury, Q. Zeng, Z. Chen, E. Mauceli, N. Hacohen, A.

Gnirke, N. Rhind, F. di Palma, B. W. Birren, C. Nusbaum, K. Lindblad-Toh, N. Friedman, A. Regev, Full-length transcriptome assembly from RNA-Seq data without a reference genome. Nature biotechnology 29, 644-652 (2011).

78. M. A. Campbell, B. J. Haas, J. P. Hamilton, S. M. Mount, C. R. Buell, Comprehensive analysis of alternative splicing in rice and comparative analyses with Arabidopsis. BMC genomics 7, 327 (2006).

79. B. J. Haas, S. L. Salzberg, W. Zhu, M. Pertea, J. E. Allen, J. Orvis, O. White, C. R. Buell, J. R. Wortman, Automated eukaryotic gene structure annotation using EVidenceModeler and the Program to Assemble Spliced Alignments. Genome biology 9, R7 (2008).

80. R. She, J. S. Chu, K. Wang, J. Pei, N. Chen, GenBlastA: enabling BLAST to identify homologous gene sequences. Genome research 19, 143-149 (2009).

81. E. Birney, M. Clamp, R. Durbin, GeneWise and Genomewise. Genome research 14, (2004).

82. T. M. Lowe, S. R. Eddy, tRNAscan-SE: a program for improved detection of transfer RNA genes in genomic sequence. Nucleic acids research 25, 955-964 (1997).

83. S. F. Altschul, W. Gish, W. Miller, E. W. Myers, D. J. Lipman, Basic Local Alignment Search Tool. Journal of molecular biology 215, 403-410 (1990).

84. S. Griffiths-Jones, S. Moxon, M. Marshall, A. Khanna, S. R. Eddy, A. Bateman, Rfam: annotating non-coding RNAs in complete genomes. Nucleic acids research 33, 
85. A. Marchler-Bauer, S. Lu, J. B. Anderson, F. Chitsaz, M. K. Derbyshire, C. DeWeese-Scott, J. H. Fong, L. Y. Geer, R. C. Geer, N. R. Gonzales, M. Gwadz, D. I. Hurwitz, J. D. Jackson, Z. Ke, C. J. Lanczycki, F. Lu, G. H. Marchler, M. Mullokandov, M. V. Omelchenko, C. L. Robertson, J. S. Song, N. Thanki, R. A. Yamashita, D. Zhang, N. Zhang, C. Zheng, S. H. Bryant, CDD: a Conserved Domain Database for the

86. B. Boeckmann, A. Bairoch, R. Apweiler, M. C. Blatter, A. Estreicher, E. Gasteiger, M. J.

804

805

806

807

808

809

810

811

812

813

814

815

816

817 Martin, K. Michoud, C. O'Donovan, I. Phan, S. Pilbout, M. Schneider, The SWISS-PROT protein knowledgebase and its supplement TrEMBL in 2003. Nucleic acids research 31, 365-370 (2003).

87. R. L. Tatusov, D. A. Natale, I. V. Garkavtsev, T. A. Tatusova, U. T. Shankavaram, B. S. Rao, B. Kiryutin, M. Y. Galperin, N. D. Fedorova, E. V. Koonin, The COG database: new developments in phylogenetic classification of proteins from complete genomes. Nucleic acids research 29, 22-28 (2001).

88. R. D. Finn, J. Mistry, B. Schuster-Bockler, S. Griffiths-Jones, V. Hollich, T. Lassmann, S. Moxon, M. Marshall, A. Khanna, R. Durbin, S. R. Eddy, E. L. Sonnhammer, A. Bateman, Pfam: clans, web tools and services. Nucleic acids research 34, D247-251 (2006).

89. J. Mistry, R. D. Finn, S. R. Eddy, A. Bateman, M. Punta, Challenges in homology search: HMMER3 and convergent evolution of coiled-coil regions. Nucleic acids research 41, e121 (2013). 
818 90. H. Li, B. Handsaker, A. Wysoker, T. Fennell, J. Ruan, N. Homer, G. Marth, G.

819 Abecasis, R. Durbin, S. Genome Project Data Processing, The Sequence Alignment/Map format and SAMtools. Bioinformatics 25, 2078-2079 (2009).

821

91. A. McKenna, M. Hanna, E. Banks, A. Sivachenko, K. Cibulskis, A. Kernytsky, K. Toolkit: a MapReduce framework for analyzing next-generation DNA sequencing data. Genome research 20, 1297-1303 (2010).

92. P. Cingolani, A. Platts, L. Wang le, M. Coon, T. Nguyen, L. Wang, S. J. Land, X. Lu, D. polymorphisms, SnpEff: SNPs in the genome of Drosophila melanogaster strain w1118; iso-2; iso-3. Fly 6, 80-92 (2012).

93. S. Purcell, B. Neale, K. Todd-Brown, L. Thomas, M. A. Ferreira, D. Bender, J. Maller, P. Sklar, P. I. de Bakker, M. J. Daly, P. C. Sham, PLINK: a tool set for whole-genome association and population-based linkage analyses. American journal of human genetics 81, 559-575 (2007).

94. D. H. Alexander, J. Novembre, K. Lange, Fast model-based estimation of ancestry in unrelated individuals. Genome research 19, 1655-1664 (2009). Genetics Analysis across Computing Platforms. Mol Biol Evo/35, 1547-1549 (2018). studies. Nature genetics 38, 904-909 (2006). 
840 97. B. Pfeifer, U. Wittelsburger, S. E. Ramos-Onsins, M. J. Lercher, PopGenome: an

841 efficient Swiss army knife for population genomic analyses in R. Mol Biol Evol 31,

$842 \quad 1929-1936(2014)$.

84398 H. Li, R. Durbin, Inference of human population history from individual whole-genome

$844 \quad$ sequences. Nature 475, 493-496 (2011).

845 
846 Acknowledgments: We thank X. Wang, Z. Jia, Z. She, Y. Zhang, Z. Yu, W. Quan, Z.

847 Zeng and Y. Ning for sample collection, and B. Yin and J. Qi for information on

848 marine currents. Funding: L.L. is supported by the National Key R\&D Program of

849 China (No. 2018YFD0900304) and the Strategic Priority Research Program of the

850 Chinese Academy of Sciences (No. XDA23050402). A.L. is supported by the

851 Distinguished Young Scientists Research Fund of Key Laboratory of Experimental

852 Marine Biology, Chinese Academy of Sciences (No. KLEMB-DYS04) and by China

853 Postdoctoral Science Foundation (No. 2019TQ0324). A.L. and L.L. are supported by

854 Key Deployment Project of Centre for Ocean Mega-Research of Science, Chinese

855 Academy of Sciences (No. COMS2019Q06). L.L. is also supported by the National

856 Natural Science Foundation of China (No. 31572620) and the Technology and the

857 Modern Agro-industry Technology Research System (No. CARS-49). Author

858 contributions: L.L., G.Z. and X.G. conceived the study and participated in final data 859 analysis, interpretation and manuscript writing. A.L. carried out data analysis and 860 drafted the manuscript. H.D., A.L., H.C., X.L. and H.Z. contributed to the selective 861 sweep analysis. A.L., Z.Z., K.Z. and C.W. collected and sampled oyster specimens. 862 A.L. and W.W. produced the $\mathrm{F}_{1}$ progeny. A.L., L.L., X.G. and G.Z. revised the 863 manuscript. All authors approved the manuscript for publication. Competing 864 interests: The authors declare no competing interests. Data and materials 865 availability: The genome, whole-genome re-sequencing and transcriptome datasets 866 were deposited in the Sequence Read Archive (SRA) database under the accession 867 number PRJNA715058. 


\section{Figures}
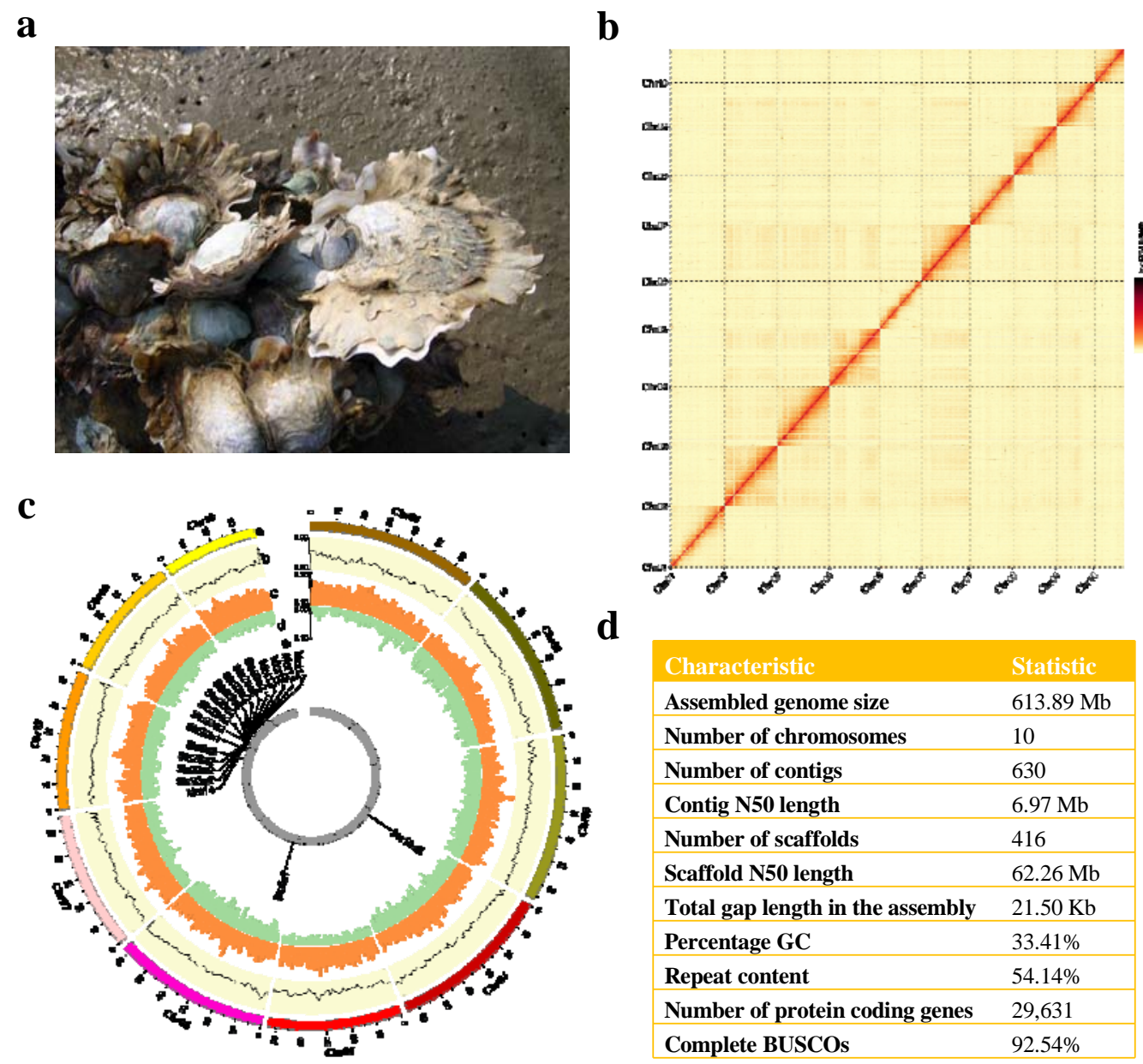

Fig. 1 | Chromosome-level assembly of the estuarine oyster Crassostrea ariakensis genome. a, Estuarine oyster (photograph by Lumin Qian). b, Hi-C interaction heatmap showing 10 chromosomes of the estuarine oyster. c, CIRCOS plot showing 10 chromosomes (a), the distribution of GC content (b), transposable elements (c), coding sequences (d), and duplicated gene cluster of the solute carrier families showing selection signals (e, also see Fig. 4). d, Summary statistics of the genome assembly. 

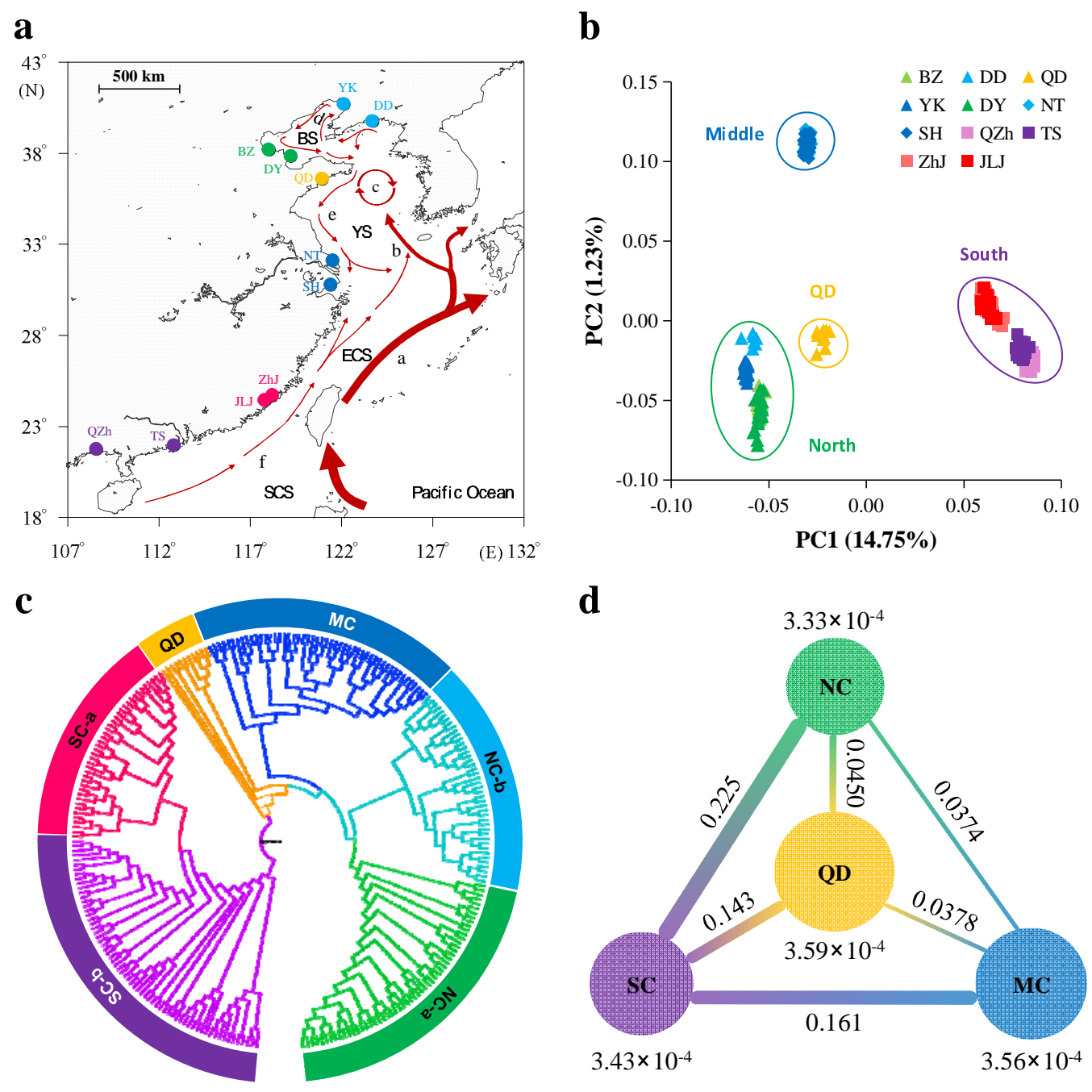

Fig. 2 | Geographic distribution and genetic differentiation of the estuarine oyster populations. a, Sampling locations of 264 resequenced wild oysters from 11 estuaries along the coast of China. The arrowed curves represent ocean currents in summer. a: Kuroshio Current, b: Yellow Sea Warm Current, c: Yellow Sea Cold Water Mass, d: Bohai Sea Circulation, e: China Coastal Current, f: South China Sea Warm Current. SCS: South China Sea, ECS: East China Sea, YS: Yellow Sea, BS: Bohai Sea. DD: Dandong, YK: Yingkou, BZ: Binzhou, DY: Dongying, QD: Qingdao, NT: Nantong, SH: Shanghai, JLJ: Jiulongjiang, ZhJ: Zhangjiang, TS: Taishan, QZh: Qinzhou. b, Plots of principal components 1 and 2 of 264 resequenced oysters based on whole-genome data. c, Phylogenetic tree of estuarine oysters inferred from genome-wide SNPs by the neighbour-joining (NJ) method. NC-a: northern China, including BZ and DY; NC-b: northern China including DD and YK; MC: middle China including NT and SH; SC-a: southern China including JLJ and ZhJ; and SC-b: southern China including TS and QZh. d, Nucleotide diversity ( $\pi$, under or above the circles) and genetic divergence ( $F_{\mathrm{ST}}$, between populations) among four populations. 
$\mathbf{a}$

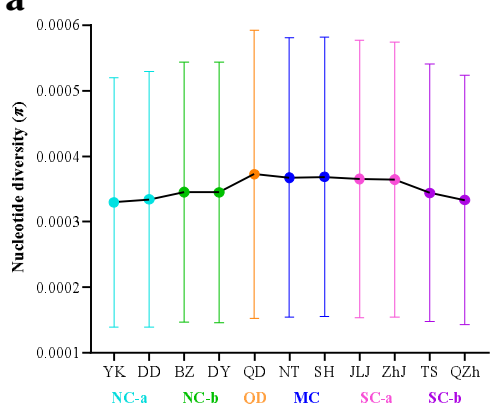

c

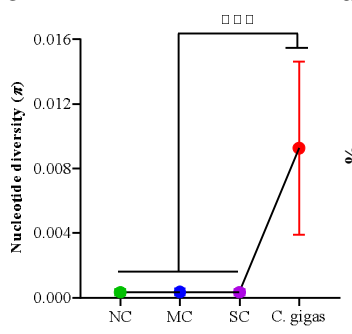

d

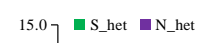

b

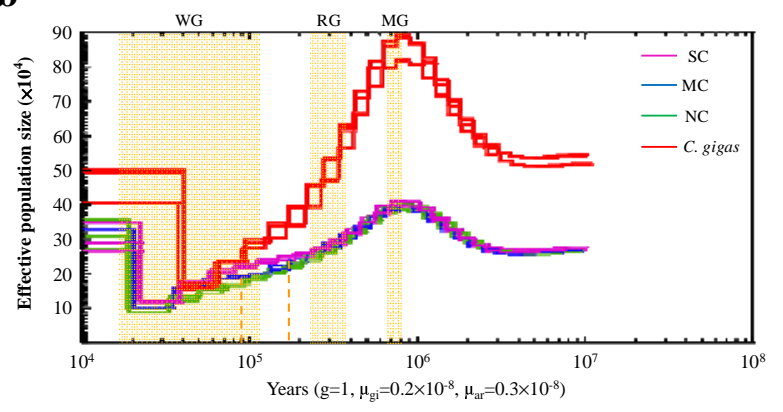

e

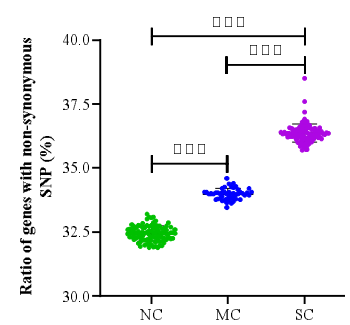

Fig. 3 | Effects of gene flow, historical glaciation and natural selection on diversity and differentiation of the estuarine oyster. a, Nucleotide diversity $(\pi)$ in 11 geographic populations along the coast of China. $\mathbf{b}$, Demographic histories of three populations of the estuarine oyster (ar) (SC, MC and NC), and Pacific oyster (C. gigas or gi) inferred by Pairwise Sequentially Markovian Coalescent. Periods of the Mindel glaciation (MG, 0.68 0.80 mya), Riss glaciation ( $\mathrm{MG}, 0.24 \sim 0.37$ mya) and Würm glaciation (WG, 10,000 120,000 years ago) were shaded by yellow. c, Nucleotide diversity $(\pi)$ of three populations of the estuarine oyster, and Pacific oyster (C. gigas). d, The ratio of SNPs that are highly heterozygous only in northern (N_het) or only in southern (S_het) populations across 10 chromosomes. e, The ratio of genes with non-synonymous SNPs in three oyster populations. Asterisks indicate significant difference $(* * * p<0.001)$. 


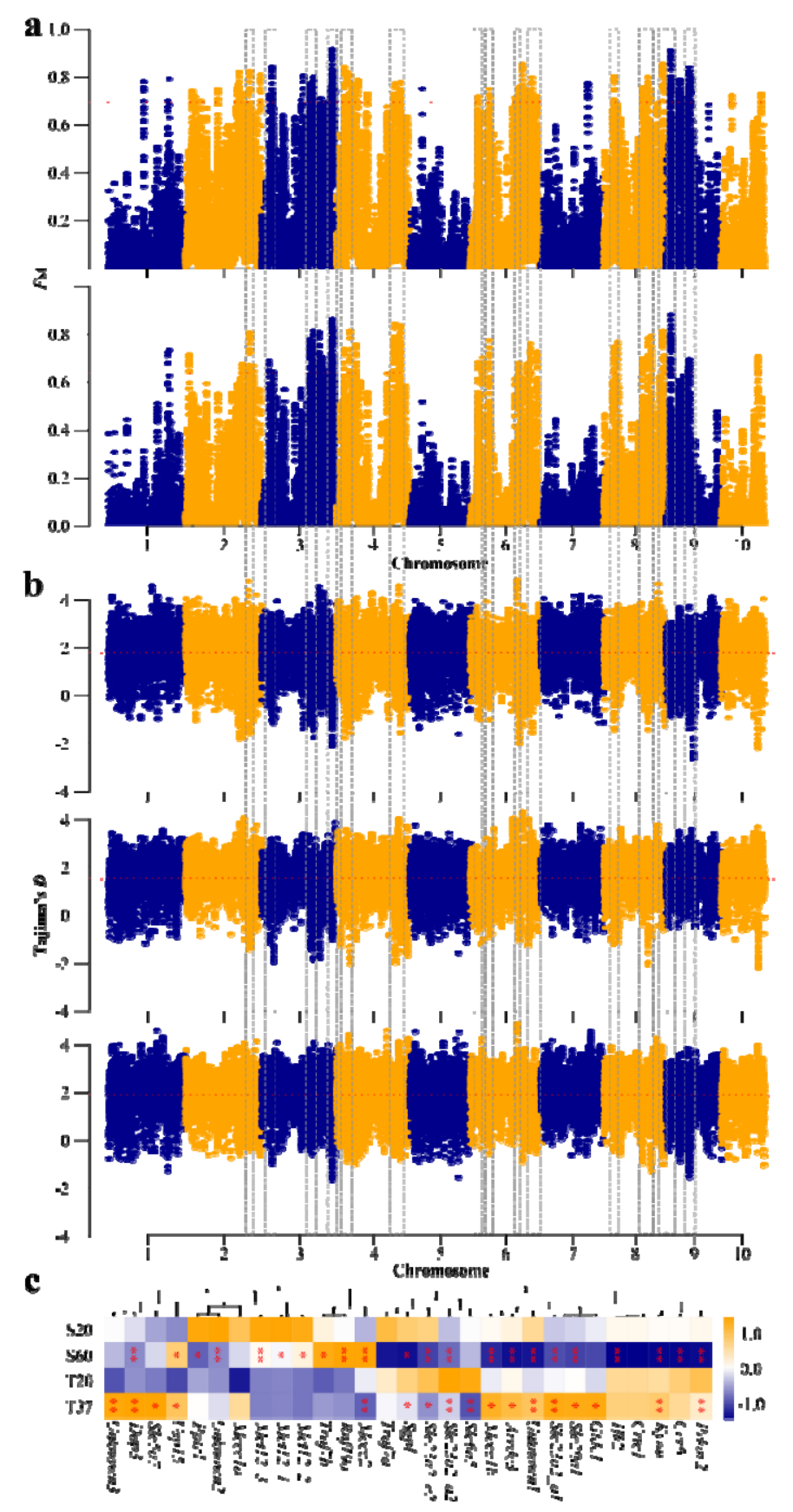

Fig. 4 | Genome-wide distribution of selective sweep signals and transcriptional responses of associated genes to thermal and salinity challenges in the estuarine oyster. a, Global $F_{\mathrm{ST}}$ values (top $1 \%$, red lines) between two population pairs: northern vs. southern (up) and middle vs. southern (bottom). b, Global Tajima's $D$ values in northern (up), middle (middle) and southern (bottom) populations. c, Expression of genes under selection exposed to thermal (6 hours at $37 \square$ ) and high-salinity ( 7 days at $60 \%$ ) challenges. Asterisks indicate significant difference $(*$ $p<0.05$, ** $p<0.01)$. 

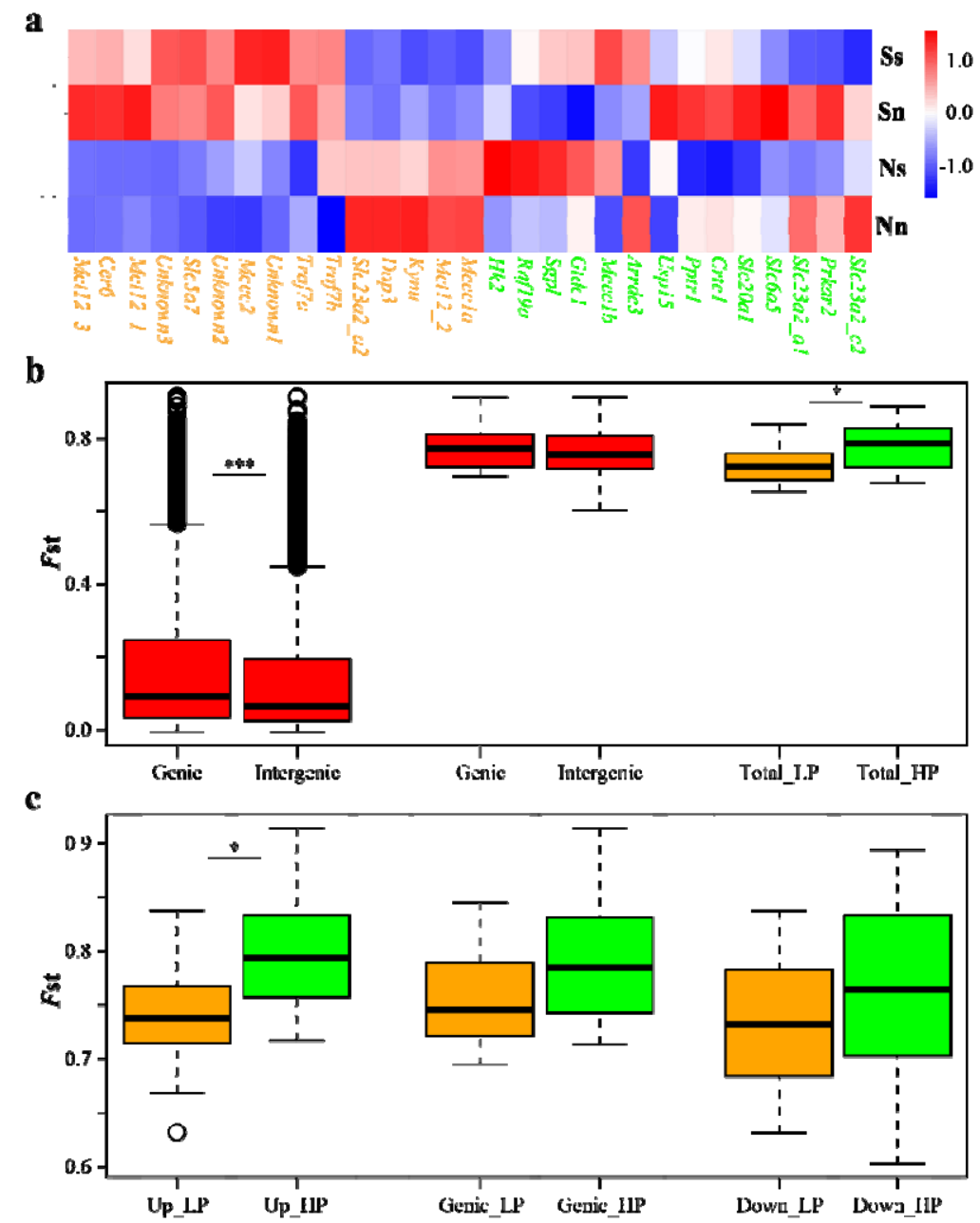

Fig. 5 | Transcriptional and genomic divergence of genes with selection signals in the estuarine oyster. a, Expression level of genes under selection in northern $(\mathrm{N})$ and southern (S) populations (capital letters) acclimated to northern (n) and southern (s) environments (lowercase letters), showing low plasticity (LP, orange) and high plasticity (HP, green) genes. b, Genetic divergence $\left(F_{\mathrm{ST}}\right)$ for genic and intergenic regions at genome level (left) and for 29 genes under selection (middle), and for LP and HP genes in both genic and intergenic regions (Total, right). c, Genetic divergence $\left(F_{\mathrm{ST}}\right)$ for intergenic [Up: upstream (left) and Down: downstream (right)] and genic (middle) regions of LP and HP genes. Asterisks indicate significant difference ${ }^{*} p<$ $0.05, * * * p<0.001)$. 


\title{
Supplementary Material for
}

\section{Genome of the estuarine oyster provides insights into climate impact and adaptive plasticity}

\author{
Subtitle: Insights into climate impact and adaptive plasticity
}

Ao Li, He Dai, Ximing Guo, Ziyan Zhang, Kexin Zhang, Chaogang Wang, Wei Wang, Hongju Chen, Xumin Li, Hongkun Zheng, Guofan Zhang* and Li Li*

*Corresponding author. Email: $\underline{\text { lili@qdio.ac.cn and gzhang@qdio.ac.cn }}$

The file includes:

Figs. S1 to S16

Other Supplementary Material for this manuscript includes the following:

Table S1-9

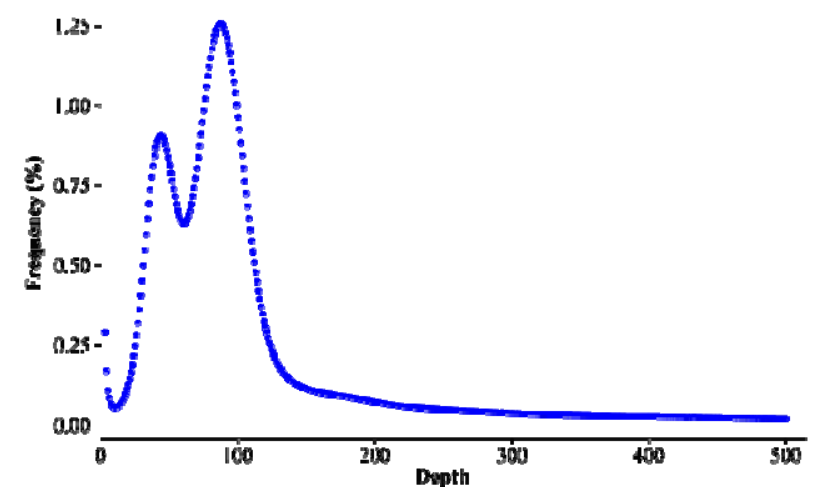

Fig. S1 | Distribution of 21-mer frequency in the sequencing reads used to estimate genome size. 


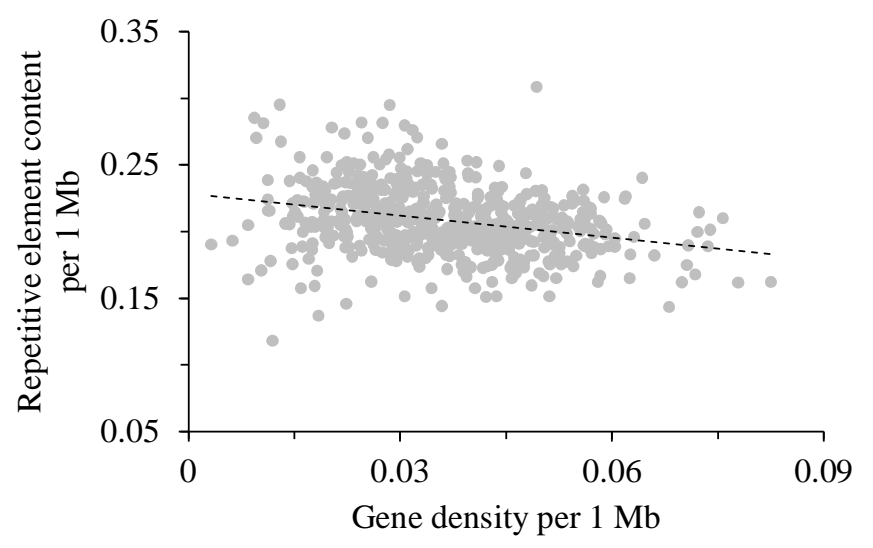

Fig. S2 | Negative correlation between gene density and repetitive element content per $1 \mathrm{Mb}$ across the genome of estuarine oyster.

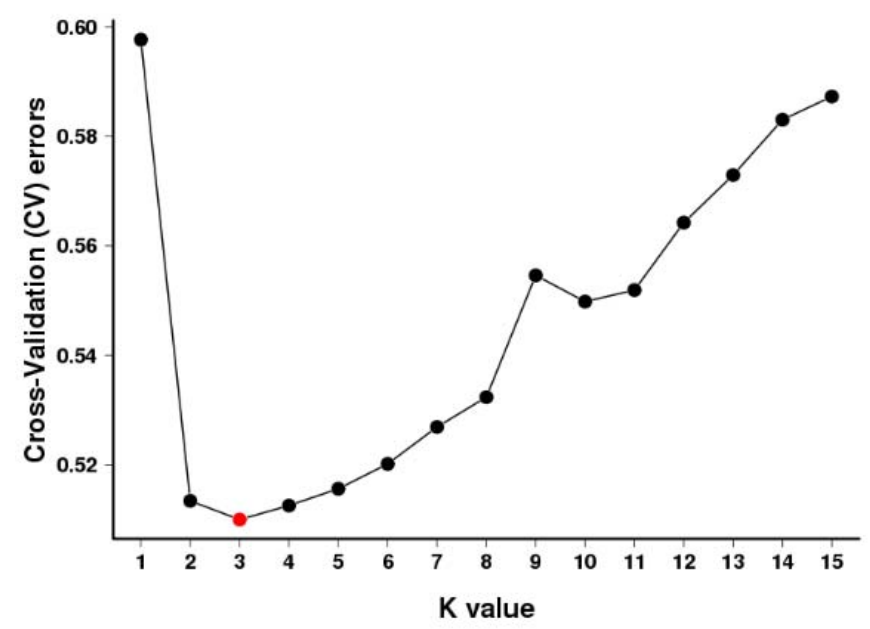

Fig. S3 | Cross-validation error plot for populations of the estuarine oyster. 


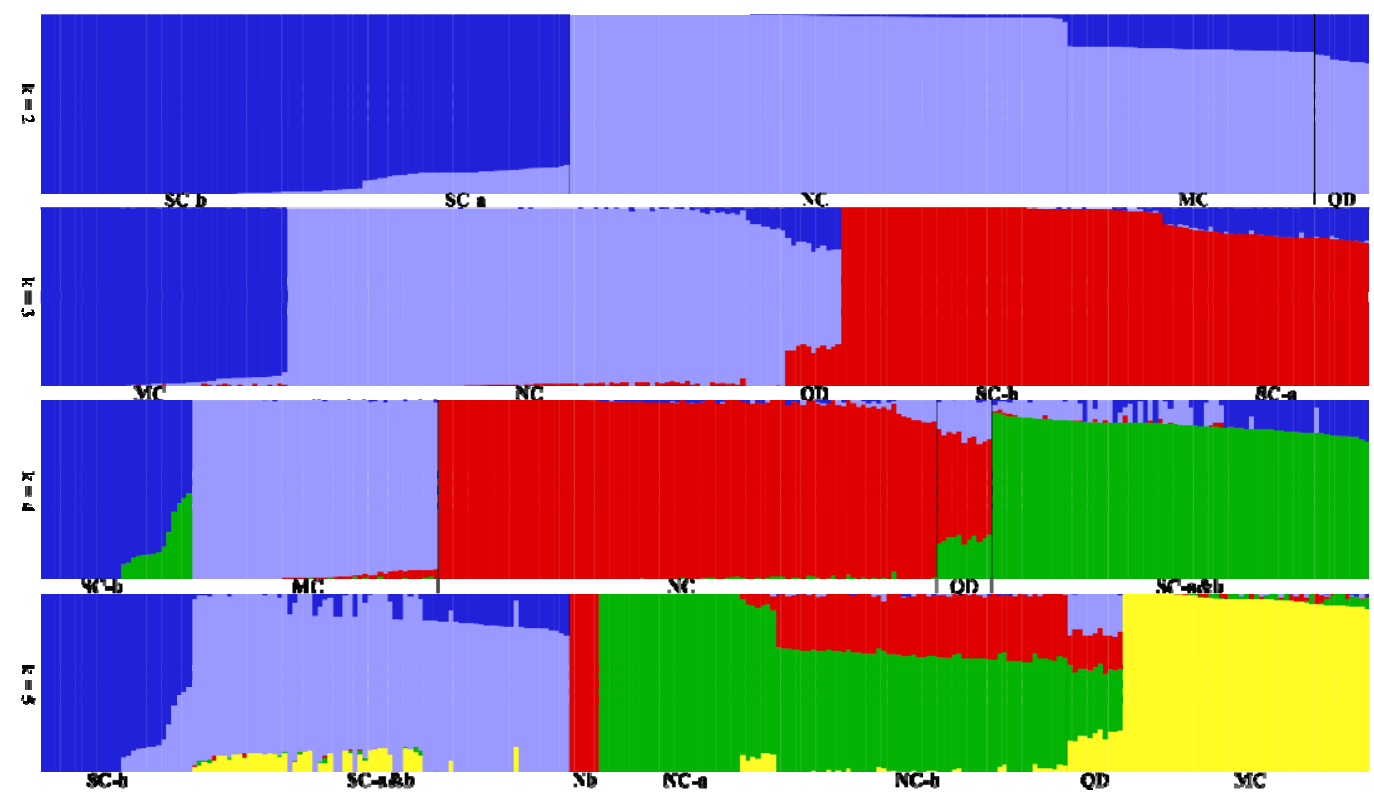

Fig. S4 | Model-based clustering analysis of estuarine oyster populations with ADMIXTURE under different number of groups $(K=2,3,4$ and 5). SC: southern China. NC: northern China (Nb indicates NC-b). MC: middle China. QD: Qingdao population.

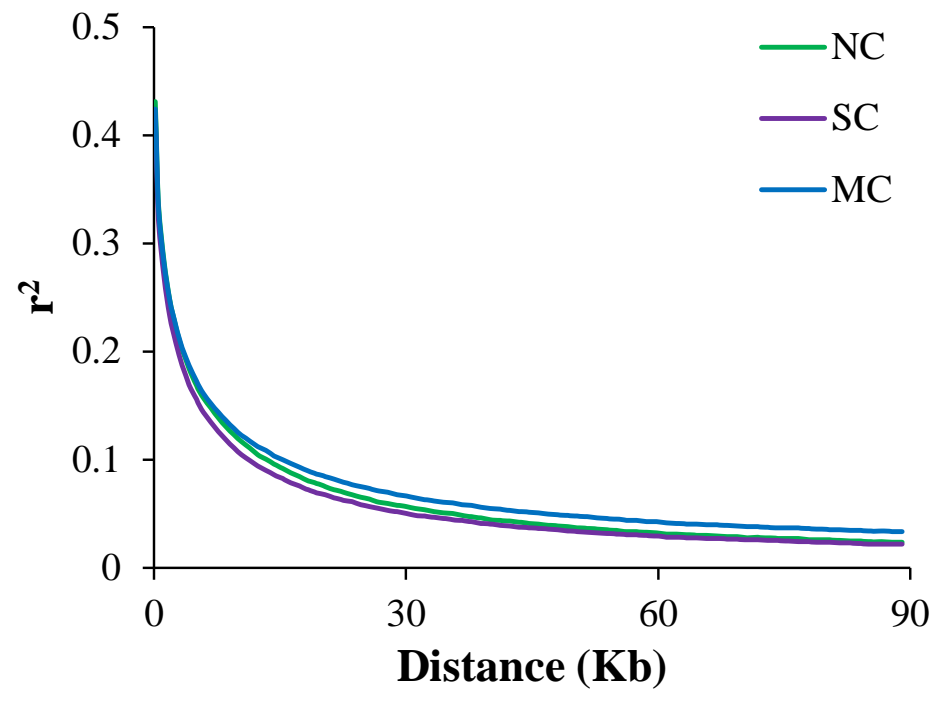

Fig. S5 | Decay of linkage disequilibrium in northern (NC), middle (MC) and southern (SC) populations of the estuarine oyster.

Fig. S6 | Dynamics of average monthly sea surface temperature (SST) and ocean currents along Chinese coastlines. Data for SST and ocean currents were downloaded from MODIS (Jan 2003 to Dec 2019) and OSCAR (Jan 2001 to Dec 2019), respectively. 


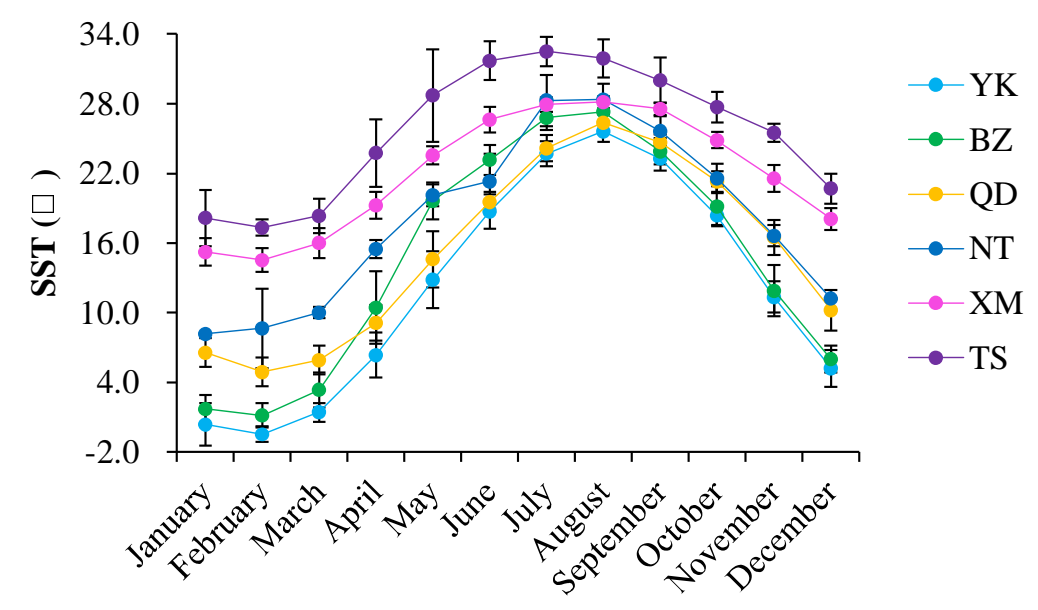

Fig. S7 | Monthly average sea surface temperature (SST) from satellite remote sensing data of six sampling sites from each of six subpopulations during 2000 to 2017. 


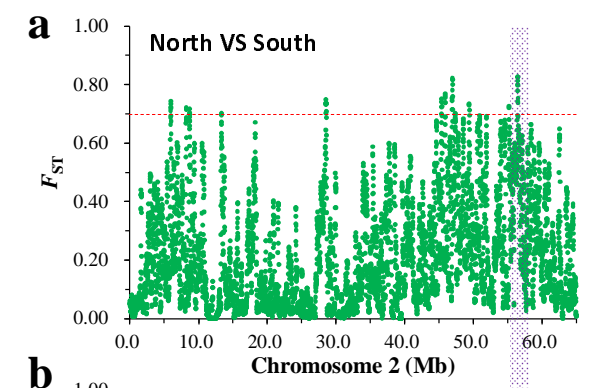

b
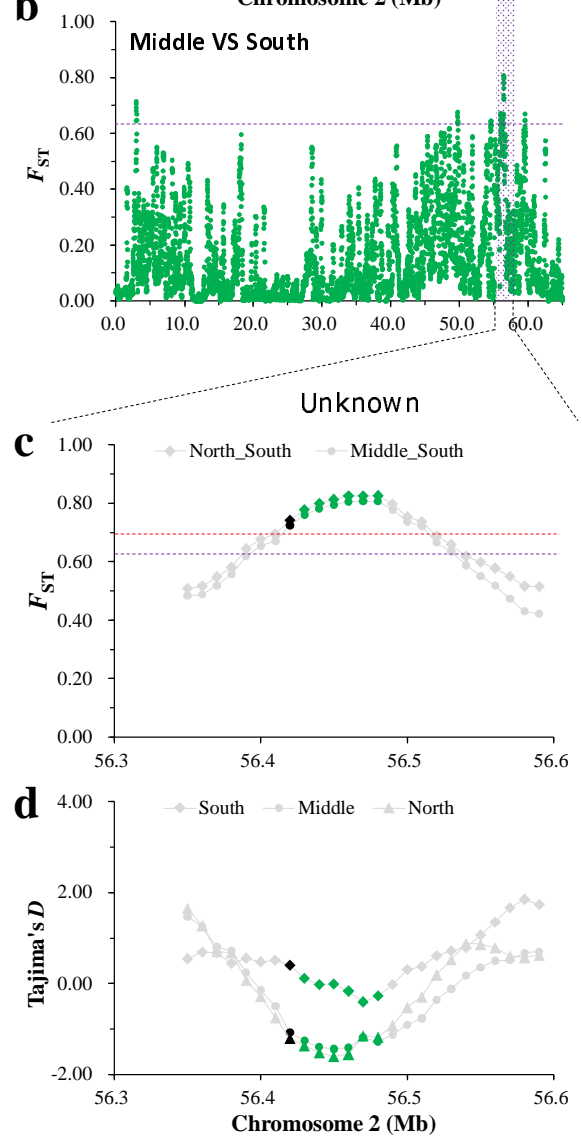

Fig. S8 | Signatures of selection along chromosome 2 in northern, middle and southern populations of the estuarine oyster. $\mathbf{a}$ and $\mathbf{b}$, Selective sweep signatures identified by top $1 \% F_{\mathrm{ST}}$ value (dash lines) between northern and southern (a), and between middle and southern populations $(\mathbf{b})$. c, $F_{\mathrm{ST}}$ values surrounding selective regions om chromosome 2 between population pairs. d, Tajima's $D$ surrounding selective regions on chromosome 2 in northern, middle and southern populations of $C$. ariakensis. Noncoding regions were marked with black, and coding regions were green. 


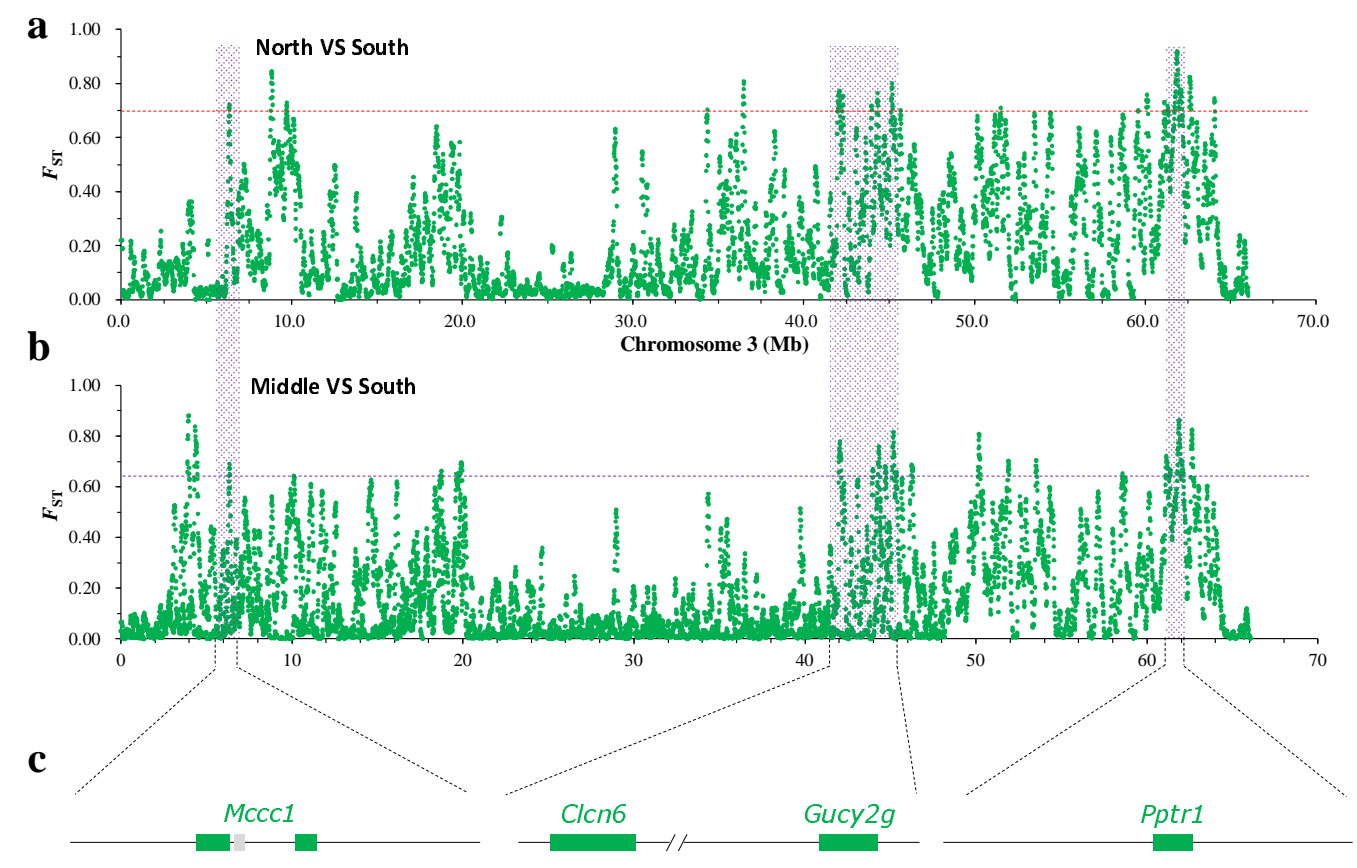

d
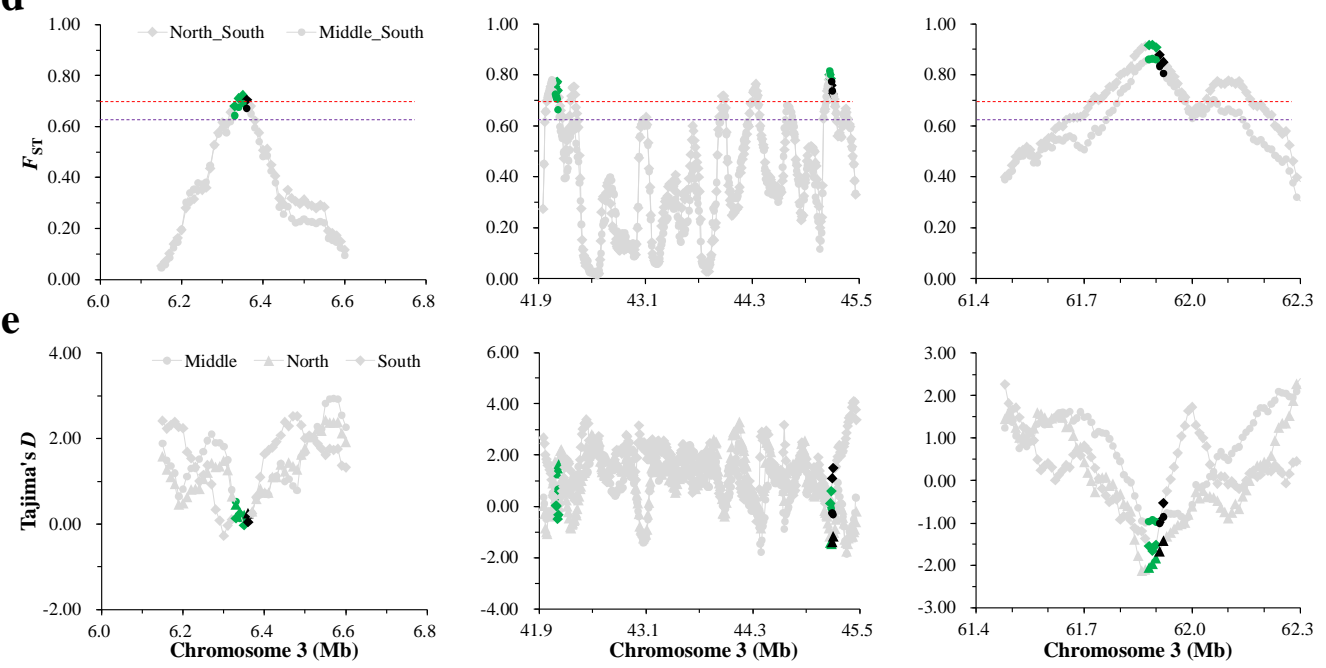

Fig. S9 | Signatures of selection along the chromosome 3 in northern, middle and southern populations of the estuarine oyster. $\mathbf{a}$ and $\mathbf{b}$, Selective sweep signatures identified by top $1 \% F_{\mathrm{ST}}$ value (dash lines) between northern and southern (a), and between middle and southern populations (b). c, Models of annotated genes located within selective regions along chromosome 3. d, $F_{\mathrm{ST}}$ values surrounding selective regions om chromosome 3 between population pairs. e, Tajima's $D$ surrounding selective regions on chromosome 3 in northern, middle and southern populations. Noncoding regions were marked with black, and coding regions were green. 


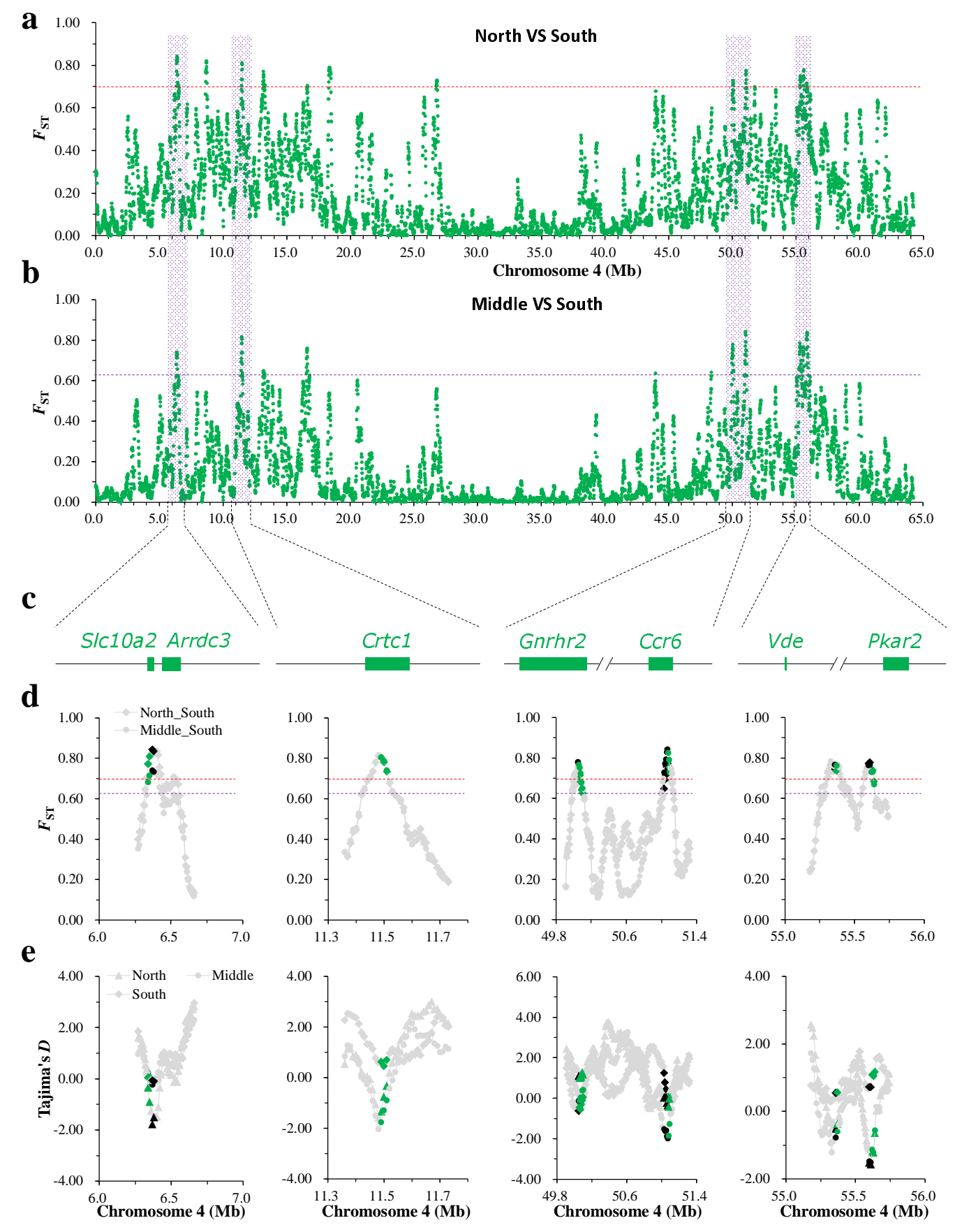

Fig. S10 | Signatures of selection along chromosome 4 in northern, middle and southern populations of the estuarine oyster. $\mathbf{a}$ and $\mathbf{b}$, Selective sweep signatures identified by top $1 \% \quad F_{\mathrm{ST}}$ value (dash lines) between northern and southern populations (a), and between middle and southern populations (b). c, Models of annotated genes located within selective regions along chromosome 4 . d, $F_{\mathrm{ST}}$ values surrounding selective regions om chromosome 4 between population pairs. e, Tajima's $D$ surrounding selective regions on chromosome 4 in northern, middle and southern populations. Noncoding regions were marked with black, and coding regions were green. 


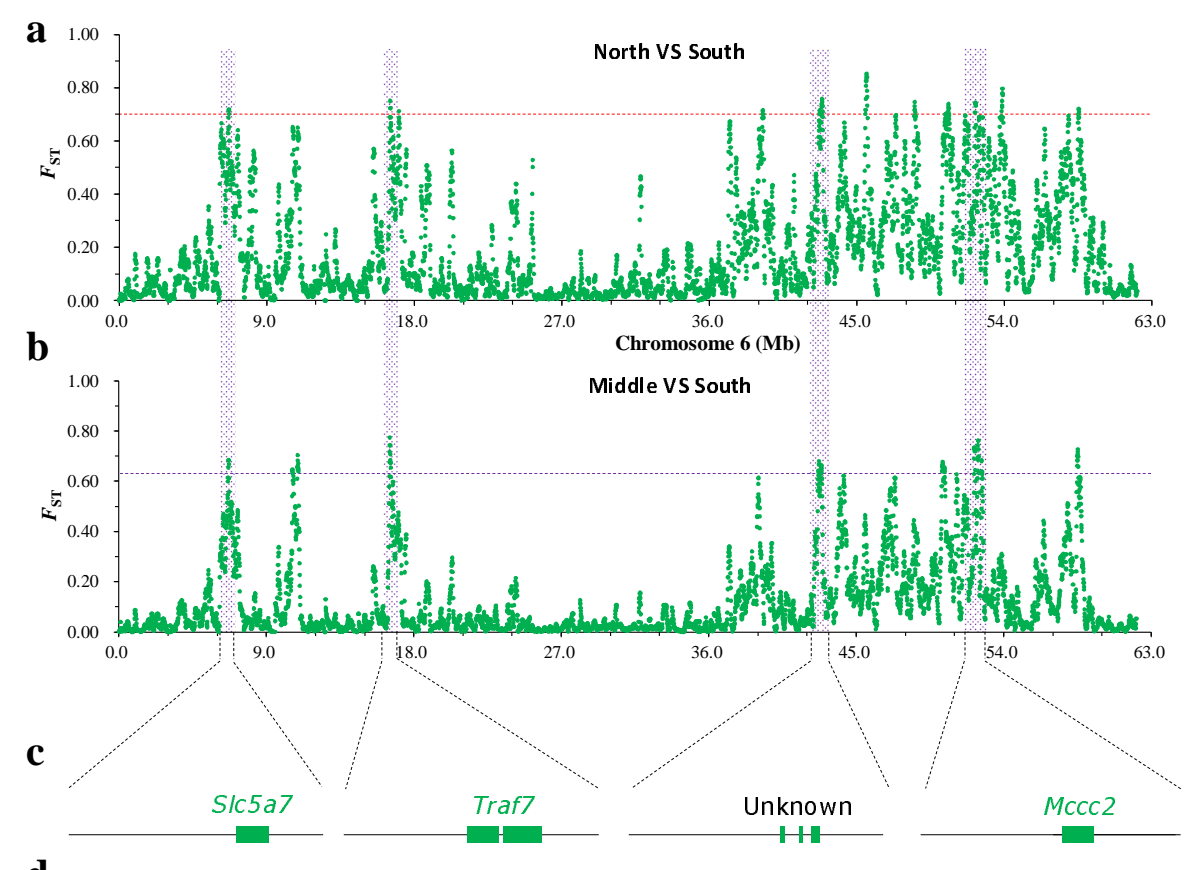

d
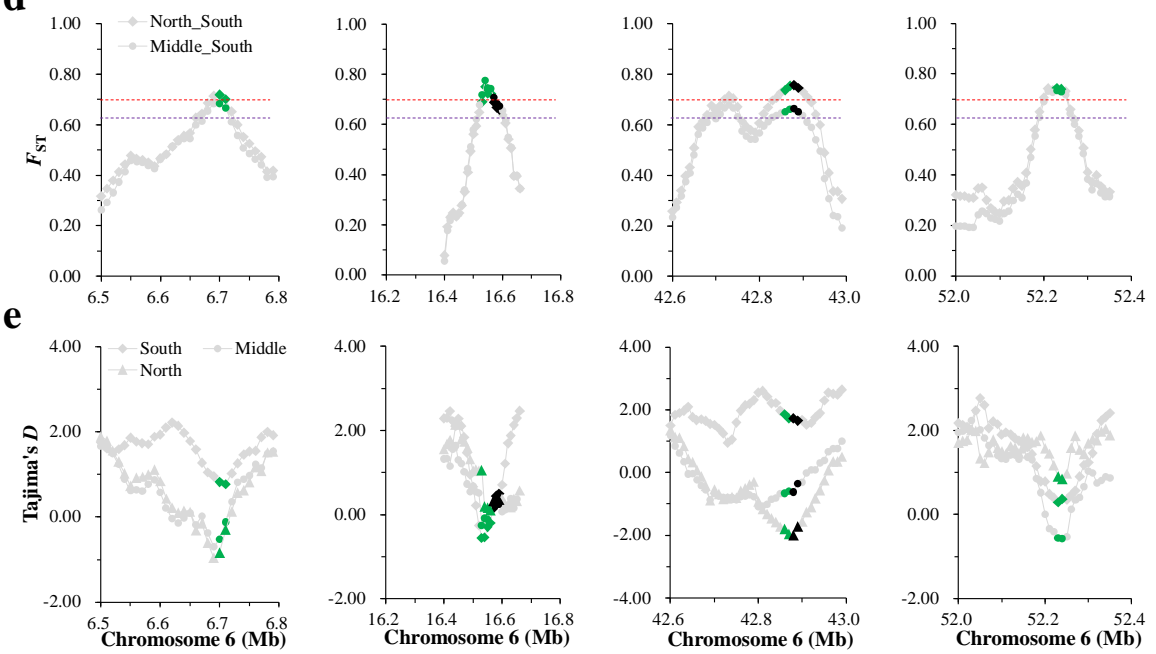

Fig. S11 | Signatures of selection along chromosome 6 in northern, middle and southern populations of the estuarine oyster. $\mathbf{a}$ and $\mathbf{b}$, Selective sweep signatures identified by top $1 \% F_{\mathrm{ST}}$ value (dash lines) between northern and southern (a), and between middle and southern populations (b). c, Models of annotated genes located within selective regions along chromosome $6 . \mathbf{d}, F_{\mathrm{ST}}$ values surrounding selective regions om chromosome 6 between population pairs. e, Tajima's $D$ surrounding selective regions on chromosome 6 in northern, middle and southern populations. Noncoding regions were marked with black, and coding regions were green. 


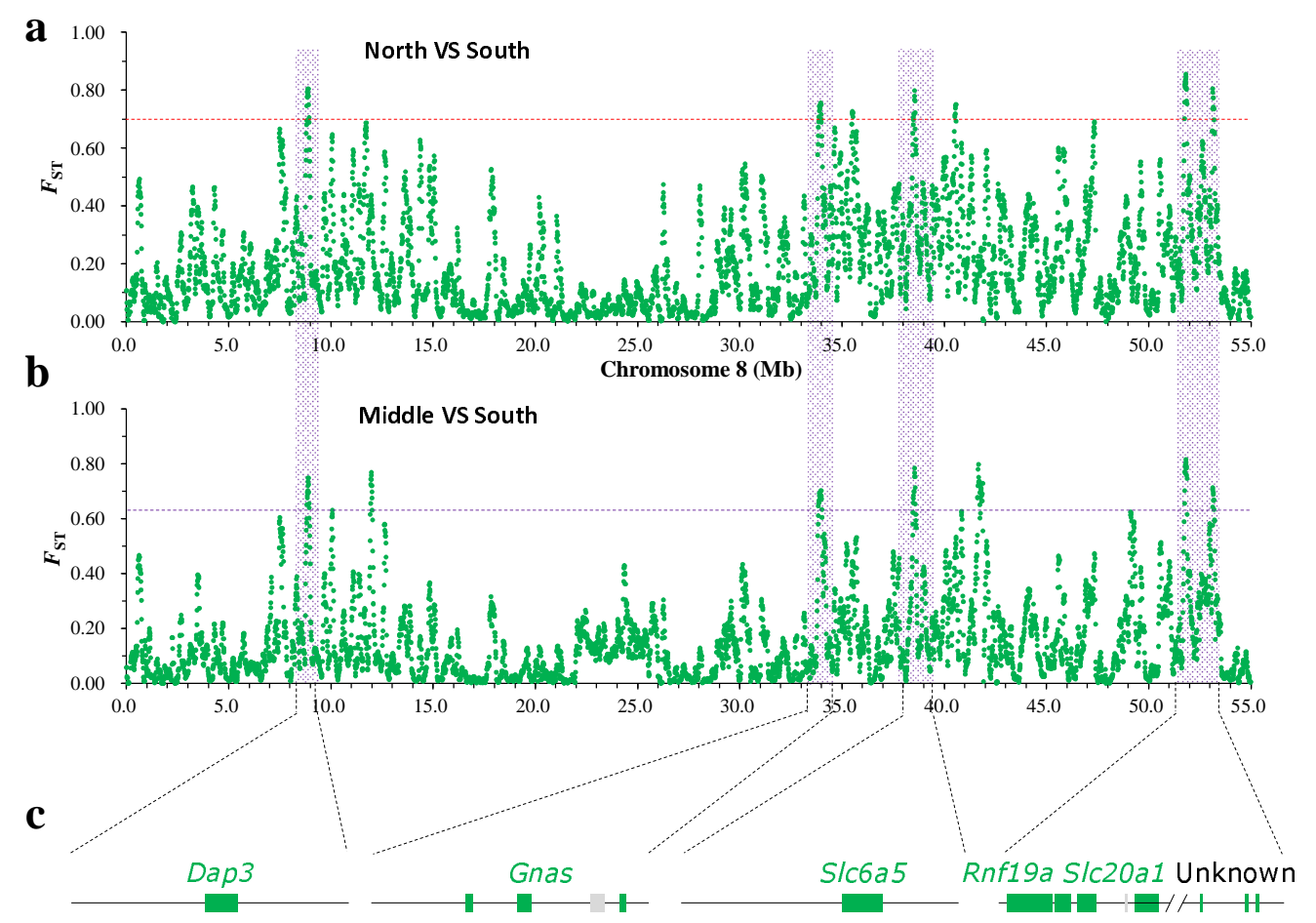

d
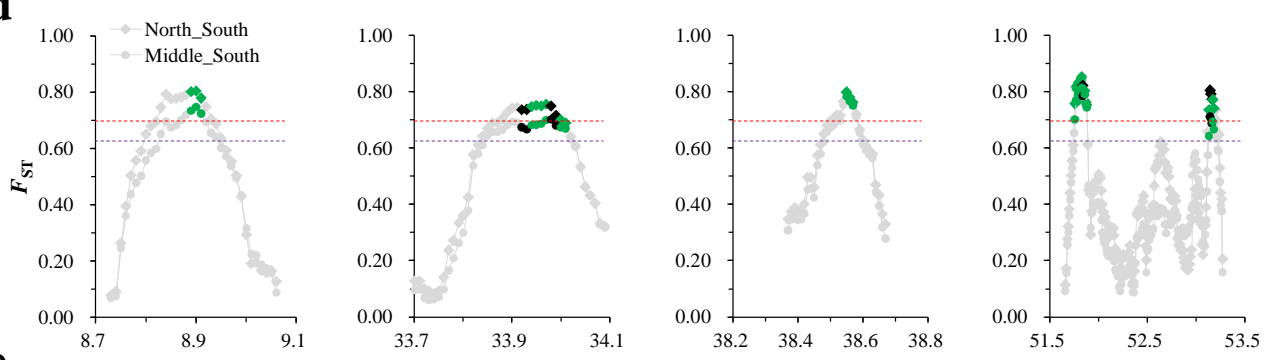

$\mathbf{e}$
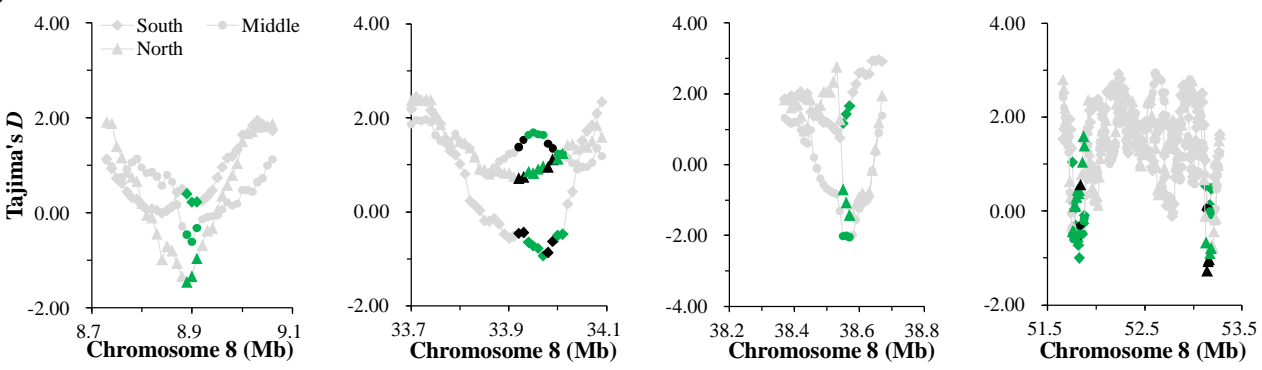

Fig. S12 | Signatures of selection along chromosome 8 in northern, middle and southern populations of the estuarine oyster. $\mathbf{a}$ and $\mathbf{b}$, Selective sweep signatures identified by top $1 \% F_{\mathrm{ST}}$ value (dash lines) between northern and southern (a), and between middle and southern populations (b). c, Models of annotated genes located within selective regions along chromosome $8 . \mathbf{d}, F_{\mathrm{ST}}$ values surrounding selective regions om chromosome 8 between population pairs. e, Tajima's $D$ surrounding selective regions on chromosome 8 in northern, middle and southern populations. Noncoding regions were marked with black, and coding regions were green. 

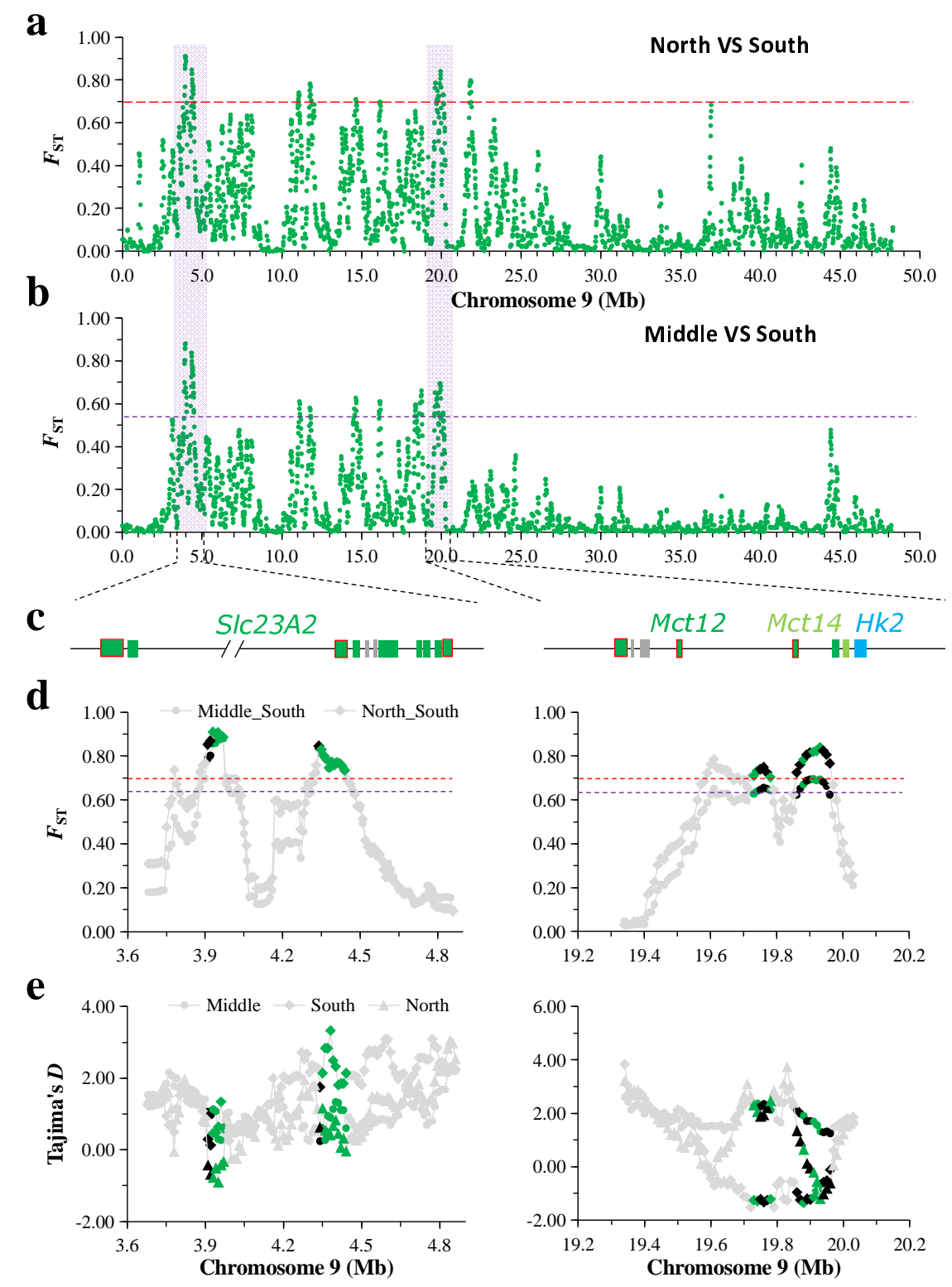

Fig. S13 | Signatures of selection along chromosome 9 in northern, middle and southern populations of the estuarine oyster. $\mathbf{a}$ and $\mathbf{b}$, Selective sweep signatures identified by top $1 \% F_{\mathrm{ST}}$ value (dash lines) between northern and southern (a), and between middle and southern populations (b). c, Models of annotated genes located within selective regions along chromosome 9 . Models with red lines are responsive to temperature and salinity challenges. d, $F_{\mathrm{ST}}$ values surrounding selective regions om chromosome 9 between population pairs. e, Tajima's $D$ surrounding selective regions on chromosome 9 in northern, middle and southern populations. Noncoding regions were marked with black, and coding regions were green. 


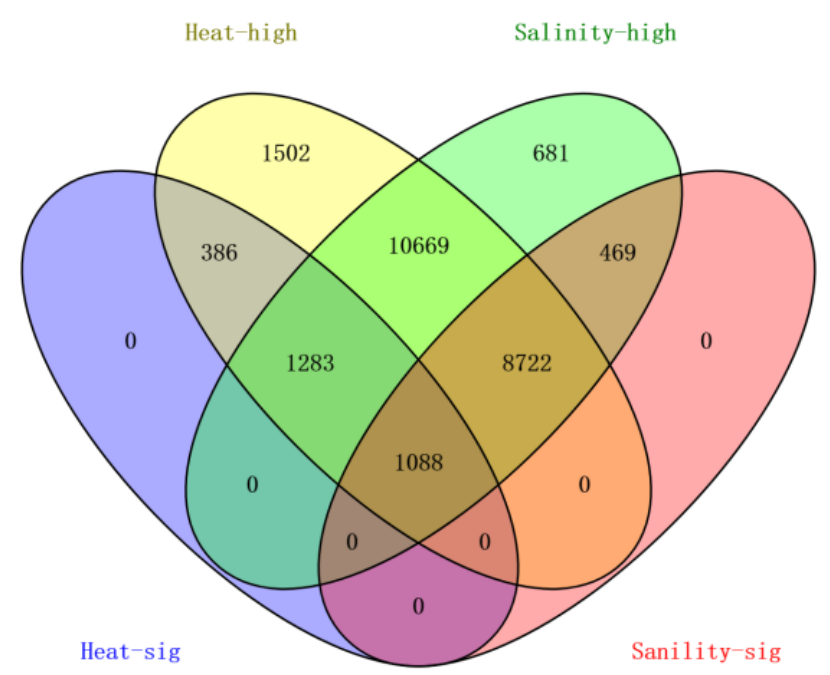

Fig. S14 | Venn diagram of genes expressed in response to high temperature and high salinity stresses in the estuarine oyster. Heat-high and salinity-high indicate genes expressed under elevated temperature (6 hours under $37 \square$ seawater) and high salinity (7 days under 60\% seawater) excluding genes with low expression. Heat-sig and salinity-sig indicate genes significantly up- or down-regulated in response to high-temperature and high-salinity stresses.

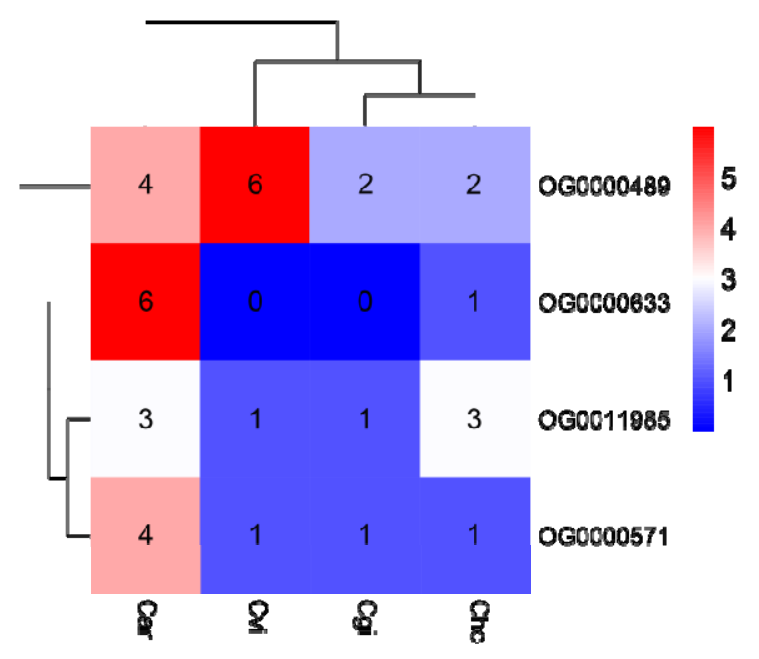

Fig. S15 | Copy numbers of orthogroups of Slc23a2 and Mct12 gene families in four Crassostrea oyster species. OG0000633: uric acid transporter, OG0011985 and OG0000489: purine permease, OG0000571: purine efflux pump. Cvi: C. virginica, Car: C. ariakensis, Cho: C. hongkongensis and Cgi: C. gigas. 


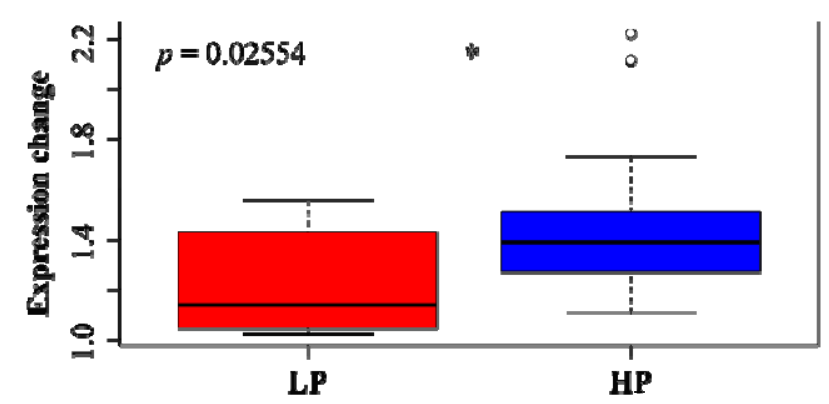

Fig. S16 | Expression changes of estuarine oyster genes showing low plasticity (LP) and high plasticity (HP) when reciprocally transplanted to non-native habitats. 